\title{
The Hypoxia Response Pathway Promotes PEP Carboxykinase Expression And Gluconeogenesis
}

Mehul Vora $^{1^{*}}$, Stephanie M. Pyonteck ${ }^{1^{*}}$, Tarmie L. Matlack ${ }^{1},{\text { Aparna } \text { Prashar }^{2} \text {, Nanci S. Kane }}^{1}$, Premal Shah ${ }^{2}$, and Christopher Rongo ${ }^{1,2}$

${ }^{1}$ The Waksman Institute, Rutgers The State University of New Jersey, Piscataway, New Jersey 08854, United States of America.

${ }^{2}$ The Department of Genetics, Rutgers The State University of New Jersey, Piscataway, New Jersey 08854, United States of America.

*These two authors made equal contributions.

To Whom Correspondence Should Be Addressed:

Christopher Rongo

Waksman Institute

Rutgers University

190 Frelinghuysen Rd.

Piscataway, NJ 08854

Phone (848) 445-0955

Fax (732) 445-5735

Email: crongo@waksman.rutgers.edu

Running Title: Hypoxia Response Pathway 


\section{ABSTRACT}

Actively dividing cells, including some cancers, rely on aerobic glycolysis rather than oxidative phosphorylation to generate energy, a phenomenon termed "the Warburg effect ${ }^{1}$." Constitutive activation of the Hypoxia Inducible Factor (HIF-1), a transcription factor known for mediating an adaptive response to oxygen deprivation (hypoxia), is a hallmark of the Warburg effect ${ }^{2}$. HIF-1 is thought to promote glycolysis and suppress oxidative phosphorylation. Here, we show instead that HIF-1 can promote gluconeogenesis. Using a multiomics approach, we determined the genomic, transcriptomic, and metabolomic landscapes regulated by constitutively active HIF-1 in C. elegans. We performed RNA-seq and ChIP-seq under aerobic conditions in mutants lacking EGL-9, a key negative regulator of HIF-1, and then integrated these approaches to identify over a hundred genes directly and functionally upregulated by HIF-1. We show that HIF-1 directly promotes the expression of PCK-1, a PEP carboxykinase that is a ratelimiting mediator of gluconeogenesis ${ }^{3}$. This activation of PCK-1 by HIF-1 promotes survival in response to both oxidative and hypoxic stress. Our work is the first to identify functional direct targets of HIF-1 in vivo, and it describes the first complete metabolome induced by constitutive HIF-1 activation in any organism. 


\section{MAIN TEXT}

Metazoans respond to hypoxia using a conserved hypoxia response pathway that mediates an adaptive response. Oxygen is sensed by a prolyl hydroxylase (EGL-9 in C. elegans), which negatively regulates Hypoxia Inducible Factor (HIF-1 in C. elegans), the pathway transcriptional effector (Fig. 1a). When hypoxia ensues, EGL-9 is inhibited, and HIF-1 becomes active. In disorders involving acute hypoxia, tissues are damaged by energy deprivation and oxidative stress $^{2,4}$. The hypoxia response pathway, when activated by hypoxia, minimizes such damage; however, long-term adaptations and tissue remodeling triggered by the hypoxia response pathway itself can also cause damage ${ }^{5,6}$. The pathway can also be activated to promote anaerobic glycolysis (i.e., the Warburg effect) even under aerobic conditions, including in some cancers, in dividing stem cells, in the proliferation of activated T lymphocytes, during endometrial decidualization, and presumably in patients receiving prolyl hydroxylase inhibitors to treat anemia associated with chronic kidney disease ${ }^{1,7-17}$. HIF-1 clearly upregulates enzymes that mediate anaerobic glycolysis, but our understanding of the complete adaptive response mobilized by pathway activation has largely focused on gene expression changes in cultured cells $^{18-21}$. We therefore set our goal on a complete characterization of the adaptive response induced by pathway activation in a whole organism context by taking advantage of the genetic model system $C$. elegans. These nematodes employ single orthologs of the conserved hypoxia response pathway, with null mutants in hif-1 and egl-9 being viable but showing altered sensitivity to hypoxic stress ${ }^{22-29}$. HIF-1 is constitutively active in egl-9 mutants, which we have used here in a combined genomic, transcriptomic, and metabolomic approach to obtain a holistic and functional description of HIF-1 activation in an intact animal.

\section{A Functional HIF-1::GFP Transgene}

To identify sites in the genome bound by HIF-1, we introduced a genomic clone ${ }^{30}$ containing a hif-1::gfp chimeric gene expressed from the hif-1 promoter into the germline genome of hif1(ia4) null mutants (Extended Data Fig. 1a,b). Quantitative mRNA measurements of this genome-integrated odls131[hif-1::gfp] transgene indicated that it is expressed at levels similar to that of endogenous hif-1 (Extended Data Fig. 1c-e). Under aerobic conditions, HIF-1 is rapidly turned over through the action of EGL-9, which uses oxygen to hydroxylate key proline residues on HIF-1. VHL-1 then ubiquitinates hydroxylated HIF-1, targeting it for degradation by the proteasome $^{23}$. By contrast, HIF-1 is stable in egl-9(sa307) null mutants. We observed little detectable fluorescence from hif-1(ia4); odls131[hif-1::gfp] animals under aerobic conditions (Fig. 1b) and nuclear HIF-1::GFP fluorescence in essentially all tissues in egl-9 mutants (Fig. 
1c). The odls131[hif-1::gfp] transgene accurately substituted for endogenous hif-1 with regard to the regulation of two established transcriptional targets (Extended Data Fig. 1f,g), egg laying (Extended Data Fig. 1h), and survival under hypoxic stress (Extended Data Fig. 1i) ${ }^{25,26,31-33 .}$ Taken together, these results show that odls131[hif-1::gfp] is functional and expresses tagged hif- 1 to levels similar to that of endogenous hif-1, providing a tool to identify genomic binding sites under physiological conditions.

\section{Identifying Direct HIF-1 Targets}

To identify genes directly regulated by HIF-1, we first performed ChIP-seq on L4-stage egl9(sa307); hif-1(ia4); odls131[hif-1::gfp] animals, which have activated HIF-1 under aerobic conditions, using anti-GFP antibodies to precipitate DNA bound by HIF-1::GFP followed by highthroughput sequencing to identify peaks of reads corresponding to HIF-1 binding sites. We identified 604 peaks (Supplemental Table 1), nearly all of which fell within 500 bps of the transcriptional start site (TSS) or stop codon of a known gene (Extended Data Fig. 2a,b), as well as regions enriched for the hypoxia response element (HRE) known to bind to human HIF1 ${ }^{34,35}$ (Extended Data Fig. 2c).

The nearest neighboring gene to a ChIP-seq peak is often not the direct target regulated by the transcription factor bound at that peak. We therefore performed RNA-seq to analyze differential gene expression due to HIF-1 activation, using these data to identify HIF-1-regulated genes near HIF-1 binding sites. We examined transcriptomes of L4-stage animals under aerobic conditions in genotypes in which HIF-1 is active, including egl-9(sa307) mutants and egl-9(sa307); hif-1(ia4); odls131[hif-1::gfp] mutants, as well as inactive, including wild type and egl-9(sa307); hif-1(ia4) double mutants (Extended Data Fig. 3a). Multidimensional Scaling analysis showed tight clustering within four biological replicates, with a clear separation between genotypes in which HIF-1 is active versus inactive (Extended Data Fig. 3b). We compared the resulting transcriptional profiles (FDR $<0.01$; Supplemental Table 2) in four distinct pairwise combinations (active vs. inactive), deeming the intersection of those comparisons as highstringency HIF-1-regulated genes (Extended Data Fig. 3a,c,d).

Direct targets of HIF-1 should (1) have a nearby HIF-1 binding site, and (2) be differentially expressed when HIF-1 is active. We adapted the BETA algorithm for $C$. elegans to integratively analyze our ChIP-seq and RNA-seq data ${ }^{36}$. Using a 15-kb window and an FDR<0.01, we identified 267 HIF-1 binding sites flanking 145 direct target genes (Fig. 1d; Extended Data Fig. 3e; Supplemental Table 3). Every identified direct target was exclusively upregulated rather than downregulated when HIF-1 was active, consistent with HIF-1 
functioning as a transcriptional activator (Fig. 1e). Motif analysis of the direct targets using MEME-suite ${ }^{37}$ showed enrichment for the conserved HRE with a preference for " $A$ " at position 15 (Fig. 1f). BETA identified nhr-57 and cysl-2 (Fig. 1g), two known HIF-1 targets, further validating our approach $25,26,31,32$. About half (53\%) of the HIF-1 targets associated with a single binding site, and the magnitude of expression for targets loosely correlated with their number of binding sites (Fig. 1g). Interestingly, the genes egl-9 and rhy-1, two key negative regulators of HIF- $1^{25}$, contained some of the highest numbers of HIF-1 binding sites (4 and 8 , respectively) and were dramatically upregulated by HIF-1 (10-fold and 76-fold, respectively), indicating a strong negative feedback loop and highlighting the importance of modulating the pathway itself as part of the response (Extended Data Fig. 3f).

\section{Characterizing HIF-1 Binding Sites}

To determine if the HIF-1 binding sites for these targets are functional, we employed a transgenic approach. We examined one novel target, pck-1 (PEP carboxykinase), associated with a single HIF-1 binding site, and one established target, rhy-1, associated with multiple binding sites. We generated fluorescent Venus transcriptional reporter transgenes containing genomic sequences encoding the HIF-1 binding site and the TSS (Fig. 2a; Extended Data Fig. 4a) fused to the sequence for Venus. In the case of $r h y-1$, our transgene only included the closest HIF-1 ChIP-seq peak upstream of the TSS. To quantify expression, we introduced into

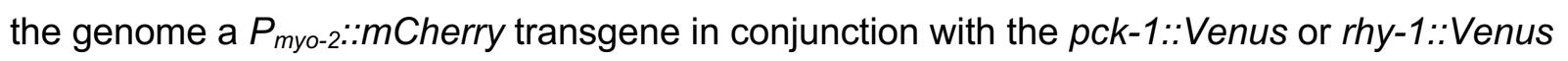
transgenes. As myo-2 expression did not vary in our RNA-seq data sets, we normalized Venus expression to mCherry expression in the pharynx. Transgenic animals for both reporters had elevated levels of Venus fluorescence when HIF-1 was activated in egl-9 mutants relative to wild type and egl-9; hif-1 double mutants, where is HIF-1 is inactive (Fig. 2b-g; Extended Data Fig. 4b). We also generated versions of the transgenes in which either the complete sequence encoding the HIF-1 ChIP-seq peak was removed ( $\Delta$ ChIP), the 6-bp core HRE in the peak was deleted $(\triangle \mathrm{HRE})$, or only a minimal promoter sequence was present (Fig. 2a; Extended Data Fig. 4a). Removal of the full sequence encoding the ChIP-seq peak or the HRE alone was sufficient to reduce reporter expression in egl-9 mutants to levels similar to that of the full-length promoter in wild type or in egl-9; hif-1 double mutants (Fig. 2h; Extended Data Fig. 4b). These data show that HREs are required in vivo for HIF-1 to regulate target gene expression.

We reasoned that HIF-1 might share target genes with other stress response pathways such that other transcription factors might bind near or in HIF-1 binding sites. In addition, published ChIP-seq studies have identified High Occupancy Target (HOT) sites where 
occupancy by multiple (>15) transcription factors occurs; it is unclear whether HOT sites represent functional binding sites or simply "sticky" regions of the genome that lend to false signals within ChIP-seq data ${ }^{38,39}$. We surveyed the known binding sites of all transcription factors within the ModENCODE/ModERN database for L4 stage animals and determined which of the 55 factors also bound in or near HIF-1 sites. An examination of all 604 ChIP-seq peak sequences revealed the vast majority of them to be in or near known HOT sites (Extended Data Fig. 4c). By contrast, less than half of the 267 functional ChIP-seq peaks identified using RNAseq data and BETA analysis were at HOT sites, suggesting many HOT sites might not be functional. Histograms of both sets of peaks (Fig. 2i) revealed three populations of HIF-1 binding sites: low occupancy (1-4 other factors bound), medium occupancy (5-20 other factors bound), and high occupancy (21 or more factors bound). Filtering by BETA caused a large drop in the number of high occupancy sites but only a subtle drop in low and medium occupancy sites, suggesting that the definition of HOT sites might need to be revisited. We observed enrichment for AHA-1 binding sites near HIF-1 sites (Fig. 2j; Extended Data Fig. 4d), as expected for a factor that acts as a HIF-1 dimerization partner ${ }^{23}$. We also observed enrichment for SKN-1 sites near HIF-1 sites, the $C$. elegans Nrf2 ortholog that promotes an antioxidant response ${ }^{40}$, suggesting shared targets between the hypoxia and antioxidant responses. By contrast, PQM-1 sites were underrepresented near HIF-1 sites, consistent with the increased hypoxia survival observed in pqm-1 mutants ${ }^{41}$. We also note that, depending on the transcription factor, shared targets might be co-regulated, regulated synergistically, or regulated antagonistically.

\section{HIF-1 Reprograms Metabolism}

To determine the types of genes regulated by HIF-1, we performed a Gene Ontology (GO) term analysis. For the 145 direct targets upregulated by HIF-1, we observed enrichment of GO terms for glycolysis, gluconeogenesis, amino acid metabolism, sulfur oxidation, fatty acid betaoxidation, and oxidation-reduction metabolism (Fig. 3a). The remaining targets that were indirectly upregulated by HIF-1 were enriched for deoxyribonucleotide metabolism and innate immunity (Extended Data Fig 5a). The increased expression of innate immunity genes could explain changes in host-pathogen interaction observed in hypoxia response pathway mutants $^{42,43}$. The targets indirectly downregulated during HIF-1 activation were enriched for GO terms for protein dephosphorylation, multicellular development, transcriptional regulation, organelle organization and biogenesis, signal transduction, and nucleic acid metabolism (Extended Data Fig 5b). Taken together, these results suggest that HIF-1 reprograms 
metabolism by directly binding to the promoters of key metabolic pathway enzymes, promoting their expression.

To confirm that activated HIF-1 reprograms metabolism, we used a metabolomics approach to determine if the transcriptionally upregulated pathways were more populated with metabolites from those pathways. We used liquid chromatography and mass spectrometry (LCMS) to obtain metabolomic profiles of L4 stage wild type and egl-9(sa307) mutants under aerobic conditions, examining 558 metabolites. We observed differences $(p<0.05)$ in the levels for 175 metabolites (Supplemental Table 4). HIF-1 activation led to elevated levels of various amino acids, carbohydrates, lipids, and nucleotides, suggesting increased flux through multiple metabolic pathways (Fig. 3b).

To examine metabolite levels in the context of specific biochemical pathways, we mapped wild type versus egl-9 mutant metabolome data against corresponding RNA-seq data for enzymes that catalyze reactions involving each specific metabolite. It is well established that HIF-1 activation in mammals promotes glycolysis by upregulating several key enzymes in the pathway ${ }^{43,44}$. Consistent with these mammalian studies, we found that HIF-1 directly promoted the expression of multiple key glycolysis enzymes and increased metabolite population of this pathway (Fig. 3c), highlighting a conserved function of HIF-1 activation between nematodes and mammals. Glycolysis generates pyruvate, which enters the tricarboxylic acid (TCA) cycle to generate NADH under aerobic conditions. Oxygen is required to oxidize that NADH and thereby generate ATP; thus, we would expect metabolite flux in the TCA cycle to be reduced when HIF-1 is activated.

As expected, most TCA cycle enzymes and metabolites are unchanged in egl-9 mutants (Fig. 3d). However, egl-9 mutants had elevated levels of $m d h-1$ (malate dehydrogenase) and Idh-1 (lactate dehydrogenase), as well as associated metabolites malate, pyruvate, and lactate (Fig. 3e). Elevated malate and malate dehydrogenase are indicative of flux through the glyoxylate cycle, a variation of the TCA cycle used in some organisms to convert citrate to oxaloacetate to feed the gluconeogenesis pathway.

We also observed indicators of increased gluconeogenesis (Fig. 3c). As previously discussed, HIF-1 directly and dramatically promotes the expression of $p c k-1$ (PEP carboxykinase), which converts oxaloacetate (OA) to phosphoenolpyruvate (PEP), a key ratelimiting step specific to gluconeogenesis. In addition to regenerating glucose, gluconeogenesis provides substrates for the pentose phosphate pathway (PPP), which generates reducing equivalent NADPH, 5-carbon sugars (e.g., ribose-5-phosphate used to synthesize nucleic acids), and erythose-4-phosphate (used to synthesize aromatic amino acids). We observed an 
increase in PPP metabolites in egl-9 mutants relative to wild type (Fig. 3f), although none of the key enzymes within the PPP were direct targets of HIF-1, suggesting that increased PPP flux is an indirect effect of HIF-1 activation of gluconeogenesis.

With elevated PPP flux, we would expect that downstream pathways that utilize NADPH to be active, with enrichment of associated metabolites in egl-9 mutants compared to wild type. For example, NADPH generated from PPP serves as reducing equivalent for fatty acid synthesis, and both synthesized and dietary fatty acids are attached to glycerol-3-phosphate (G3P) for conversion to storage lipids. Consistent with metabolic pathway, we observed decreased G3P and increased levels of certain fatty acids when HIF-1 is active (Fig. 3g). Phospholipids are also synthesized from G3P, and we found that active HIF-1 increased the levels of certain phospholipid species, as well as endocannabinoid-like fatty acid ethanolamides and lysophospholipids often used as secondary signaling molecules (Fig. 3g).

NADPH also replenishes reduced glutathione, a major antioxidant for combating oxidative stress, and we observed higher levels of glutathione in egl-9 mutants relative to wild type (Fig. 3h). Indeed, many of the direct target genes most dramatically upregulated by HIF-1 lie within pathways that produces glutathione (Fig. 3h), and the catalases and superoxide dismutases that combat oxidative stress are all indirectly upregulated (Fig. 3i). Oxidative stress response pathways help fight bacterial infection in $C$. elegans, as bacterial pathogens like Pseudomonas aeruginosa produce toxins like $\mathrm{HCN}$, which mimics hypoxia, and $\mathrm{H}_{2} \mathrm{~S}^{44,45}$. We observed that HIF-1 directly promotes the expression of the entire $\mathrm{H}_{2} \mathrm{~S} / \mathrm{HCN}$ detoxification pathway (Fig. 3j), including cysl-2 (cyanoalanine synthase) and sqrd-1 (sulfide quinone oxidoreductase), which convert these toxins to polysulfides and sulfates ${ }^{44}$. Upregulation of $\mathrm{H}_{2} \mathrm{~S} / \mathrm{HCN}$ detoxification by HIF-1 is consistent with egl-9 and hif-1 mutants being resistant and sensitive, respectively, to Pseudomonas infection and sulfide/cyanide toxicity.

\section{HIF-1 and PCK-1 Are Required for Adaptive Survival to Hypoxia and Oxidative Stress}

Traditionally, HIF-1 is thought to protect against hypoxic stress by promoting anaerobic ATP synthesis, yet our multiomics analyses highlighted its promotion of the oxidative stress response. Gluconeogenesis plays a central role in promoting oxidative stress resistance through generation of NADPH and glutathione. We therefore examined null mutants for pck-1, the rate limiting enzyme of gluconeogenesis directly regulated by HIF-1. These pck-1(ok2098) mutants were viable under aerobic conditions, most likely because two other PEP carboxykinases, $p c k-2$ and $p c k-3$, compensate for baseline function. Neither pck-2 nor pck-3 showed HIF-1-dependent regulation, suggesting pck-1 is a specific HIF-1-induced isoform. We 
examined changes in oxidative stress sensitivity directly by exposing L4-stage animals to a lethal dose of paraquat, a superoxide generator. Less than half of wild-type animals survive after 15 hours of paraquat exposure. By contrast, most egl-9 mutants survived, and this survival was HIF-1- and PCK-1-dependent (Fig. 4a). PCK-1 mediates gluconeogenesis by producing PEP, and we observed elevated PEP levels when HIF-1 was activated in egl-9 mutants (Fig. 3c). Supplementation of animals with PEP was sufficient to restore survival to hif-1 and pck-1 mutants exposed to paraquat (Fig. 4a), demonstrating the importance of PCK-1 and gluconeogenesis upregulation by HIF-1 in combating oxidative stress.

Given that both hypoxia and subsequent reoxygenation result in the production of reactive oxygen species (ROS) and oxidative stress, we reasoned that HIF-1 and PCK-1 might also protect against hypoxia. We exposed L4-stage animals to $48 \mathrm{hrs}$ of hypoxia without food and measured survival after $24 \mathrm{hrs}$ of recovery at normoxia. While only half of wild-type animals survived this assay, nearly all egl-9 mutants survived, and this survival was HIF-1- and PCK-1dependent (Fig. 4b). In addition, both hif-1 and pck-1 single mutants were significantly susceptible to hypoxia, and this susceptibility was rescued by PEP supplementation (Fig. 4b,c). Although more variable by trial, we observed similar results with a hypoxia survival assay on food at higher temperature (Extended Data Fig. 6a,b). We also observed that gpd-3(ok2870) mutants, which are incapable of continuing gluconeogenesis, survived hypoxia poorly (Extended Data Fig. 6a,b). Mutations in pck-1 did not suppress the egg retention or extended lifespan phenotypes of egl-9 mutants (Extended Data Fig. 6c,d), indicating that the upregulation of PCK1 does not mediate all functions of HIF-1. Taken together, our results suggest that HIF-1 activation allows animals to survive hypoxia by primarily and directly promoting gluconeogenesis and oxidative stress resistance.

\section{Discussion}

Transcriptional effectors of stress response pathways coordinate the regulated expression of multiple target genes needed to rebalance physiological homeostasis over time and in different tissues. As an effector for both the hypoxia response pathway and the Warburg effect, HIF-1 promotes ATP synthesis by promoting glycolysis, yet the nature and role of its full array of targets in vivo is not understood. Our studies combined ChIP-seq and RNA-seq to identify a full array of direct HIF-1 targets, combined with metabolomics to understand the physiological changes resulting from their expression. We also observed changes in expression of indirect targets, which could reflect either a second phase of regulation by transcription factors elevated during the first phase or the homeostatic action of other physiological pathways responding to 
changes due to HIF-1 activation. Our metabolomics analysis confirmed that the HIF-1-mediated expression of these targets reprograms metabolism beyond simply augmenting glycolysis. Instead, we found that HIF-1 directly promotes lipid metabolism, the glyoxylate cycle, gluconeogenesis, the PPP, $\mathrm{H}_{2} \mathrm{~S} / \mathrm{HCN}$ detoxification, and glutathione synthesis, making ours the first study to combine genomic, transcriptomic, and metabolomic approaches to characterize the action of HIF-1 activation in vivo

Activation of the glyoxylate cycle was intriguing, as it allows some organisms to mobilize lipids into acetyl-CoA, which can then be converted into oxaloacetate for gluconeogenesis and/or lactic acid production. Compared to the TCA cycle, the glyoxylate cycle does not produce as much $\mathrm{NADH}$ requiring oxidation nor carbon lost as $\mathrm{CO}_{2}$, which would be advantageous under hypoxic conditions. C. elegans could use this cycle to derive energy from stored lipids under fairly anaerobic conditions when beta oxidation would not be effective. It could also be used to derive glucose from stored lipids, which could be used to generate reducing power, antioxidants, or macromolecules via the PPP. Although the glyoxylate cycle was assumed to be absent in vertebrates, more recent evidence suggests that a vertebrate version of the pathway acts in the liver ${ }^{46,47}$.

HIF-1 directly promotes the expression of the pck-1 PEP carboxykinase, a key mediator of gluconeogenesis, as well as metabolic flux through the pathway (Fig. 4d). Gluconeogenesis provides the carbon needed to make glutathione and produce NADPH reducing equivalents required to combat $\mathrm{ROS}$ and oxidative stress. Indeed, we find that $p c k-1$ mutants survive hypoxic stress as poorly as hif-1 mutants, and HIF-1 activation can offset damage by agents that cause oxidative stress. Our results highlight that the need for HIF-1 activation in mobilizing antioxidant defenses is likely as important as its role in promoting anaerobic energy production. Moreover, blocking gluconeogenesis might present an approach to reduce progression of HIF1-positive tumors. 


\section{METHODS}

\section{Generation of Transgenes and Transgenic Animals}

The GFP-tagged hif-1 transgene was obtained as a genomic fosmid from the TransgeneOme Project $^{30}$. The polyclonal stab culture was streaked out under triple selection (chloramphenicol, streptomycin, nourseothricin) and individual clones were selected for transgene construct validation by sequencing. The construct was then stably integrated into the genome of unc119(ed3) animals using microparticle bombardment ${ }^{48}$. The resulting stably integrated line odls131 was made homozygous by selecting single hermaphrodites that gave $100 \%$ Non-Unc progeny, then outcrossed four times. Subsequently, the unc-119(ed3); odls131 strain was crossed to unc-119(ed3); egl-9(sa307); hif-1(ia4) to generate the following strains used for ChIP-seq.

To generate $P_{p c k-1:}:$ Venus transgenic animals, $2816 \mathrm{bp}$ upstream of the ATG of $p c k-1$ was amplified and cloned upstream of a venus coding sequence present in pPD95.77-mVenus. Using Q5-site directed mutagenesis (Life Technologies Ltd), the $\Delta$ Peak (1084 - 912 upstream of start) and the $\Delta \mathrm{HRE}\left(1019-1003\right.$ upstream of start) were deleted to yield $P_{p c k-1(\Delta P e a k):: V e n u s}$

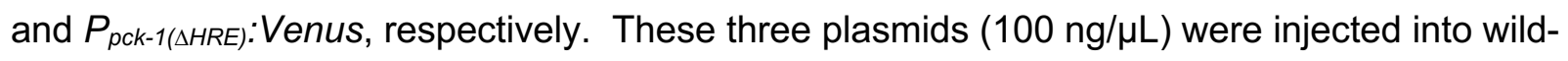
type animals at $100 \mathrm{ng} / \mu \mathrm{L}$ along with a $P_{\text {myo-2 }}:: m$ Cherry $(50 \mathrm{ng} / \mu \mathrm{L})$ co-injection marker. At least two independent lines were tested for each experiment.

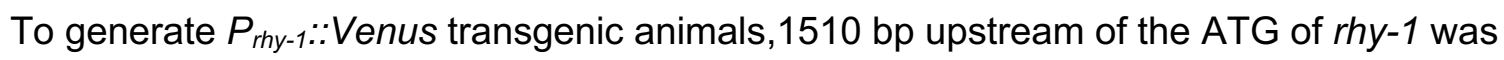
amplified and cloned upstream of a venus coding sequence as above. Q5-site directed mutagenesis (Life Technologies Ltd) was used for the removal of the $\Delta$ Peak $(1170-617 \mathrm{bp}$ upstream of start) , $\Delta \mathrm{HRE} 1$ (971 - $956 \mathrm{bp}$ upstream of start), $\Delta \mathrm{HRE} 2$ (946 - $931 \mathrm{bp}$ upstream of start) and $\min (591 \mathrm{p}$ to start) variants of the promoter. Each plasmid (100 $\mathrm{ng} / \mu \mathrm{L})$ was injected into wild-type animals at $100 \mathrm{ng} / \mu \mathrm{L}$ along with a $P_{\text {myo-2: }}: m$ Cherry $(50 \mathrm{ng} / \mu \mathrm{L})$ co-injection marker. At least two independent lines were tested for each experiment.

\section{Epifluorescence Microscopy and Image Analysis}

Fluorescent proteins were visualized in nematodes by mounting on $2 \%$ agarose pads with 10 $\mathrm{mM}$ tetramisole. All animals were synchronized by alkaline bleaching and visualized at the L4 stage. Fluorescent images of transgenic animals containing the odls131[hif-1::gfp], $P_{p c k-}$

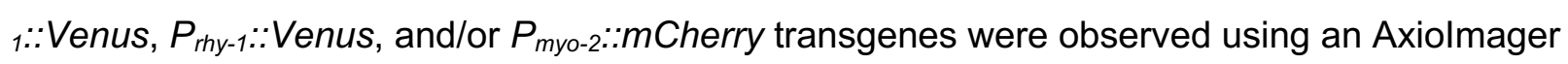
M1m (Carl Zeiss, Thornwood, NY). A 10X or 40X PlanApo objective was used to detect fluorescence. Images were acquired with an ORCA charge-coupled device camera 
(Hamamatsu, Bridgewater, NJ) by using iVision software (Biovision Technologies, Uwchlan, $\mathrm{PA}$ ). Exposure times were chosen to capture at least $95 \%$ of the dynamic range of fluorescent intensity of all samples. Images were quantified in Fiji by selecting each entire animal using the segmented line tool and measuring pixel intensity for the different channels ${ }^{49}$. All data with normal distributions were analyzed with GraphPad Prism 8 in most cases using ANOVA with Dunnett's post-hoc test correction for multiple comparisons.

\section{Quantitative RT-PCR to Assess HIF-1::GFP mRNA Expression}

Two sets of primers were used to assess the expression level of the endogenous hif- 1 as well as the odls131[hif-1::gfp] transgene. The first set (5'-ACTTGCCTGACTTTACACCTG-3' and 5'TGTTGGAATGGTTGATAATGTTGAG-3') amplified a sequence in the 3' end of all hif-1 mRNA transcripts. The second set (5'-GCTTGGACGGCTTTGTTATG-3' and 5'-

GAAGGGCTCGACCTGTTAAAT-3') amplified a sequence within the hif-1(ia4) deletion. Quantitative RT-PCR was performed on mRNA isolated from L4 stage animals for the relevant genotypes, and relative mRNA abundance was performed using act-5 as a normalization control.

\section{Characterizing Egg Laying and Egg Retention}

All genotypes were synchronized by alkaline bleaching and arrested at L1 stage overnight in M9 buffer. Synchronized genotypes were assayed 43-46 hours after reaching the $L 4$ stage, when embryos present within adult animals were counted using a dissection microscope.

\section{ChIP-seq}

ChIP-Seq was performed on L4 stage by the modERN/modEncode consortium as per their standard protocol ${ }^{38}$. Developmental synchronization was achieved by bleaching and L1 arrest. Arrested L1s were plated on NGM plates seeded with OP50 bacteria and grown for $6 \mathrm{hr}$ at $20^{\circ} \mathrm{C}$ for L1 collection. L4 stage animals were harvested, and samples were cross-linked with $2 \%$ formaldehyde for $30 \mathrm{~min}$ at room temperature and then quenched with $1 \mathrm{M}$ Tris $\mathrm{pH} 7.5$. The pelleted nematodes were subsequently flash frozen in liquid nitrogen and stored at $-80^{\circ} \mathrm{C}$. Pellets were thawed on ice and $750 \mathrm{ml}$ of FA buffer containing protease inhibitors (Roche Cat\#11697498001 Complete Protease Inhibitor Cocktail Tablet, $125 \mu 100$ mM PMSF, and 25 $\mu \mathrm{l} 1 \mathrm{M}$ DTT per $25 \mathrm{ml}$ FA buffer) was added, and samples were then transferred to a $2 \mathrm{ml}$ KONTES dounce (Kimble Chase, Vineland, NJ). Samples were dounced on ice 15 times with the small "A" pestle for two cycles with a 1 min hold between each cycle. Samples were then 
dounced 15 times with the large "B" pestle for four rounds with a 1 min hold between each cycle. Samples were then sonicated to shear chromatin into 200-800 bp DNA fragments.

For each sample, $4 \mathrm{mg}$ of protein lysate was immunoprecipitated using anti-GFP antibodies (gifts of Tony Hyman and Kevin White). GammaBind G Sepharose beads (GE Healthcare Life Sciences) were pre-washed and blocked in binding buffer and $0.1 \mathrm{mg} / \mathrm{ml} \mathrm{BSA}$. Samples were pre-cleared by adding $100 \mu \mathrm{l}$ of $50 / 50$ bead/buffer solution at $4^{\circ} \mathrm{C}$, followed by centrifugation. Samples of each replicate were removed and pooled to serve as total chromatin input. To each replicate, $15 \mu \mathrm{g}$ of antibody was added overnight at $4^{\circ} \mathrm{C}$, followed by another overnight of incubation with the bead mix. Immunoprecipitates (IPs) were washed four times with cold lysis buffer and twice with cold TE. Pellets were resuspended in elution buffer (10 mM EDTA, $1 \%$ SDS, and $50 \mathrm{mM}$ Tris- $\mathrm{HCl} \mathrm{pH} 8$ ) and incubated at $65^{\circ} \mathrm{C}$ for $10 \mathrm{~min}$. Samples were centrifuged and supernatants transferred to a fresh tube. Pellets were resuspended in $29 \% \mathrm{TE}$ and $0.67 \%$ SDS and immediately centrifuged. Elution supernatants were combined and incubated at $65^{\circ} \mathrm{C}$ with mild shaking overnight. Chromatin input samples were incubated at $60^{\circ} \mathrm{C}$ with mild shaking overnight following the addition of Proteinase $\mathrm{K}$ and SDS to final concentrations of $0.1 \mathrm{mg} / \mathrm{ml}$ and $0.01 \%$, respectively. The next day, inputs were incubated at $70^{\circ} \mathrm{C}$ for $20 \mathrm{~min}$. Proteinase $\mathrm{K}$ was added to each IP and incubated at $50^{\circ} \mathrm{C}$ for $2 \mathrm{hr}$. RNaseA was added to the chromatin input to a concentration of $0.017 \mathrm{mg} / \mathrm{ml}$ and incubated at $37^{\circ} \mathrm{C}$ for 2 hr. DNA was purified with MinElute columns (QIAGEN, Valencia, CA), eluting in $13 \mu$ (elution buffer provided with MinElute kit). An additional $48 \mu \mathrm{l}$ EB was added to input samples after purification. Samples were stored at $-20^{\circ} \mathrm{C}$.

The enriched DNA fragments and input control (genomic DNA from the same sample) for two biological replicates were used for library preparation and sequencing. Samples were libraried and multiplexed using the Ovation Ultralow DR Multiplex Systems 1-8 and 9-16 (NuGEN Technologies, San Carlos, CA) following the manufacturer's protocol, except that QIAGEN MinElute PCR purification kits were used to isolate the DNA. Briefly, $1 \mu l$ of input DNA and $10 \mu$ of IP DNA was used to prepare sequencing libraries using NuGEN Ultralow library kits. Samples were prepared according to the manufacturer's protocol. Sequencing was performed on the Illumina HiSequation 2000/2500/4000.

The Illumina sequencing data were aligned to the reference genome using the BurrowsWheeler Aligner (BWA). Data were aligned to genome version WS245. Peak regions significantly enriched in aligned reads were called by ChIP-seq processing pipeline standard for modERN/ModENCODE ${ }^{38}$. Peaks above an irreproducibility discovery rate (IDR) of $0.1 \%$ were used to generate final peak sets. 
ChIP-seq data sets are available at NIH/NCBI GEO through accession number GSE7173333.

\section{RNA-seq}

Developmentally synchronized animals were obtained by hypochlorite treatment of gravid adults and embryos hatched overnight for 15-17 hours in M9. Starvation-arrested L1s were plated on NGM plates and grown at $20^{\circ} \mathrm{C}$ until L4 stage. Total RNA was isolated from animals using Trizol (Invitrogen) combined with Bead Beater lysis in four biological replicates for each genotype. An mRNA library (single-end, 50-bp reads) was prepared for each sample/replicate using Illumina Truseq with PolyA selection (Genewiz or RUCDR). Libraries were sequenced across two lanes on an Illumina HiSeq2000 (GeneWiz) or an Illumina HiSeq 2500 in Rapid Run Mode (RUCDR). Reads were mapped to the C. elegans genome (WS245) and gene counts generated with STAR 2.5.1a. Normalization and statistical analysis on gene counts were performed with EdgeR using generalized linear model functionality and tagwise dispersion estimates. Likelihood ratio tests were conducted in a pairwise fashion between genotypes with a Benjamini and Hochberg correction. Genes were considered to be HIF-1-dependent if they were differentially expressed with an FDR $<0.01$ in the same direction (up or down) in all four of the following pairwise comparisons: (1) egl-9 vs. N2; (2) egl-9 vs. egl-9 hif-1; (3) odls131; egl-9 vs. N2; (4) odls131; egl-9 vs. egl-9 hif-1. RNA-seq data sets are available at NIH/NCBI GEO through accession number GSE173581.

\section{Identification of direct targets using BETA basic}

BETA basic was used to identify potential direct targets for HIF-1 for the WS245 annotation of the C. elegans genome ${ }^{36}$. The following parameters were used: $15 \mathrm{~kb}$ from TSS, FDR cutoff of 0.01 and one-tail KS test cutoff of 0.01. The input files consisted of .bed files of IDR thresholded peaks and differential expression $\log _{2}$ FC and FDR values for the different genotypic pairwise comparisons. The final list of targets was the intersection of these comparisons.

\section{Motif Identification and Enrichment}

The sequences for the middle of each ChIP-Seq peak (+/- $100 \mathrm{bp}$, repeat masked with $\mathrm{N})$ for were extracted from the UCSC Genome Browser and entered into the MEME-Chip tool at meme-suite. org $^{37}$. The following parameters were used: JASPA2018 Core Vertebrate Database (non-redundant), motif width from $6-15 \mathrm{bp}, 1^{\text {st }}$ order background model, STREME cutoff of 0.05 . 


\section{Identification of Transcription Factor Occupancy at HIF-1 Binding Sites}

Binding site coordinates (IDR Thresholded peaks, FDR < 0.01) for all transcription factors for which ChIP-seq was conducted at the L4 stage were obtained from the modEncode repository ${ }^{38}$. In order to determine the number of instances these peaks overlapped with the HIF-1 ChIP-seq peaks for OR3350, we developed an R script that is available at https://github.com/shahlab/hypoxia-multiomics.

\section{Metabolomics}

All genotypes were age-synchronized by alkaline bleaching, and arrested L1 larvae were plated on $100 \mathrm{~mm}$ plates containing standard NGM media with OP50. Animals were grown at $20^{\circ} \mathrm{C}$ until L4 stage, at which point they were washed into $50 \mathrm{ml}$ conical bottom tubes and allowed to settle for 15 mins. Animals were then washed with 3 x $40 \mathrm{ml}$ sterile M9 and centrifuged at 2000 rpm for 5 mins. Five hundred $\mu \mathrm{L}$ of the final pellet was transferred to a $15 \mathrm{ml}$ conical bottom tube and flash frozen in liquid $\mathrm{N}_{2}$ and stored at $-80^{\circ} \mathrm{C}$ until sent to Metabolon (Metabolon Ltd) for metabolomic processing. Samples were extracted with methanol, and each extract was divided into four fractions: two for analysis by two separate reverse phase (RP)/UPLC-MS/MS methods with positive ion mode electrospray ionization (ESI), one for analysis by RP/UPLCMS/MS with negative ion mode ESI, and one for analysis by HILIC/UPLC-MS/MS with negative ion mode ESI. Raw data was extracted and peak-identified, then compared to a library based on authenticated standards.

\section{Paraquat Survival Assay}

Paraquat sensitivity was performed according to the acute paraquat sensitivity assay described in Senchuk et al ${ }^{50}$. Animals were placed at the $\mathrm{L} 4$ stage at $20^{\circ} \mathrm{C}$ and survival was counted every hour until all animals were dead. At least 20 animals per strain per trial were used for the assays.

\section{Hypoxia Survival Assays}

For hypoxia-starvation, M9 solution was pre-equilibrated with $0.5 \% \mathrm{O}_{2}$ for at least an hour. Nematodes were grown on standard NGM plates. Age-synchronized L4 animals $(n \geq 20)$ were then collected from plates, washed with M9 buffer, and then incubated with hypoxic M9 solution in capped tubes at $20^{\circ} \mathrm{C}$ for the indicated time. After hypoxia-starvation, nematodes were collected by centrifugate and returned to standard NGM plates under normoxia for 24 hours to 
allow recovery. Survival of animals was counted by assessing movement and response to gentle touch by a platinum wire pick.

For hypoxia on plates with food, a nitrogen-displacement hypoxia chamber (BioSpherix) was pre-equilibrated to $0.5 \% \mathrm{O}_{2}$ for at least $2 \mathrm{hrs}$. Standard NGM plates were ringed with garlic extract then allowed to dry 30 minutes to prevent animals from escaping up the plate sides during hypoxic exposure ${ }^{51}$. Age-synchronized L4 animals $(n \geq 20)$ were then placed in the plate and incubated at $25^{\circ} \mathrm{C}, 0.5 \% \mathrm{O}_{2}$, for $48 \mathrm{hrs}$. Control plates were placed in $21.1 \% \mathrm{O}_{2}$ at $25^{\circ} \mathrm{C}$. At the end of $48 \mathrm{hrs}$, plates were moved to $20^{\circ} \mathrm{C}$ for $24 \mathrm{hrs}$, after which survival of animals was counted by assessing movement and response to gentle touch by a platinum wire pick.

\section{PEP Supplementation}

For the paraquat survival and hypoxia assays, nematodes were grown on standard NGM plates containing $50 \mathrm{mM}$ PEP prior to collection at the L4 stage for the assay. The same protocol was then followed as above for each assay. No PEP was included in the incubation plates, M9 solution, or recovery plates. 


\section{ACKNOWLEDGEMENTS}

We thank the TransgenOme Project for the hif-1::gfp fosmid, Peter Schweinsberg and Barth Grant for help with bomdbardment integration, Daja O'Bryant and Hazel Schubert for assistance in establishing bioinformatic pipelines, and Michelle Kudron and Valerie Reinke for their assistance with the ChIP-seq. This work was supported by an NIH grant (R01GM101972) to CR and a New Jersey Commission on Spinal Cord Research Postdoctoral Fellowship (CSCR13FEL001) to SMP. 


\section{AUTHOR CONTRIBUTIONS}

MV, SMP, and CR designed the overall set of experiments, as well as analyzed and interpreted the data. SMP and TLM mediated the ChIP-seq and RNA-seq, whereas MV, SMP, PS, and CR analyzed the results. MV mediated and analyzed the metabolomics. MV, AP, and NSK constructed and analyzed the pck-1 promoter mutations. MV and CR conducted hypoxia and oxidative stress survival assays, as well as wrote the manuscript. 
bioRxiv preprint doi: https://doi.org/10.1101/2021.05.04.442650; this version posted May 4, 2021. The copyright holder for this preprint (which was not certified by peer review) is the author/funder. All rights reserved. No reuse allowed without permission.

Hypoxia Response Pathway

Vora et al.

\section{COMPETING INTEREST DECLARATION}

The authors declare no competing interests. 


\section{FIGURE LEGENDS}

\section{Figure 1. Identifying Genes Directly Regulated By HIF-1.}

(a) Diagram of the hypoxia response pathway in C. elegans. HIF-1 is hydroxylated by EGL-9, ubiquitinated by $\mathrm{VHL}-1$, and degraded by the proteasome. In egl-9 mutants, HIF-1 remains stable and regulates the transcription of target genes whose expression alters metabolism. (b,c) HIF-1::GFP fluorescence in the indicated genotypes. Bar indicates 30 microns. Strategy for integrating HIF-1 ChIP-seq and RNA-seq data to identify directly regulated targets. (e) Volcano plot of RNA-seq FDR values versus $\log _{2}$ fold change expression for individual genes (dots) in egl-9(sa307) mutants relative to wild type. The 145 direct targets identified by BETA are indicated with red diamonds. (f) Consensus HRE sequences identified by MEMEsuite in humans and enriched in the $C$. elegans ChIP-seq sequences identified by BETA. (g) Graph of $\log _{2}$ fold change in expression for individual genes (dots) in egl-9(sa307) mutants relative to wild type versus number of HIF-1 binding sites for those genes identified by ChIP. seq. Target genes mentioned in the text are circled and labeled. Error bars indicate SEM.

\section{Figure 2. Characterizing HIF-1 Binding Sites.}

(a) Diagram of the pck-1 locus and the different promoters used to drive Venus expression. The oval indicates HIF-1 and the black line beneath it indicates the HIF-1 binding site identified by ChIP-seq. The inverted triangle indicates the site of the HRE motif. Arrows indicate the TSS. Boxes indicate exons. The yellow arrow indicates sequences encoding the fluorescent Venus reporter. The black bar under the gene indicates sequences removed in the pck-1(ok2098) deletion. (b-g) Fluorescence images of animals expressing the indicated transgenes and with the indicated genotype. Venus fluorescence is indicated in green. Fluorescence from mCherry is red. Bar indicates 100 microns. (h) Graph of Venus/mCherry fluorescence ratios for the indicated genotype. Bar color indicates specific reporter as per panel A. Error bars indicate SEM. ${ }^{* * *} p<0.001,{ }^{*} p<0.05$ ANOVA/Bonferroni Multiple Comparison test for the indicated comparisons. (i) Violin plot of histogram counting the number of HIF-1 binding sites (ChIP-seq peaks) against the number of other transcription factors (TFs) known to bind to each site's region of the genome (within $400 \mathrm{bps}$ ). The top violin plot indicates all HIF-1 binding sites, whereas the bottom violin plot indicates only the functional HIF-1 binding sites identified by BETA. Solid line indicates median, whereas dotted lines indicate quartiles. HOT sites (peaks near the binding site of 15 or more other transcription factors) are indicated by the bracket. (j) Graph of the fold change in enrichment (log2) of each indicated transcription factor bound within 
400 bps of a HIF-1 binding site (ChIP-seq peak center). TFs with overrepresented or underrepresented binding near HIF-1 sites compared to chance $(p<0.01$ via bootstrap confidence interval) are highlighted in yellow or cyan, respectively.

\section{Figure 3. HIF-1 Reprograms Metabolism.}

(a) Enriched Gene Ontology (GO) classifications for the 145 direct HIF-1 targets. Fold enrichment and FDR for each classification and subclassification is indicated. (b) Overview of major metabolic pathways impacted by HIF-1 activation. Yellow and cyan arrows indicate pathways upregulated or downregulated, respectively, in egl-9 mutants relative to wild type. (cj) Combined heat map for metabolites (red) and the enzymes (black) and directional pathways (arrows) known to regulate their metabolism. On one side of each pathway arrow, differentially colored boxes indicate enzyme mRNA expression levels ( $\log _{2}$ fold change) in egl- 9 mutants relative to wild type; enzymes are labeled in black by their indicated $C$. elegans gene name. On the other side of each pathway arrow, differentially colored boxes indicate metabolites levels ( $\log _{2}$ fold change) in egl-9 mutants relative to wild type (and egl-9; hif-1 double mutants relative to wild type in panel $\mathrm{g}$ ); metabolites are labeled in red. Gene expression changes that exceed a $\log _{2}$ fold change of 1.5 are marked with an asterisk showing the actual $\log _{2}$ fold change value. Yellow arrow heads indicate genes with HIF-1 binding sites and showing direct regulation by HIF-1. Glycolysis and gluconeogenesis are graphed together, in opposite direction, as they share multiple metabolites and enzymes.

\section{Figure 4. HIF-1 Targets Required for Adaptive Survival.}

(a) Percent of L4-stage animals to survive paraquat $\left(200 \mathrm{mM}, 15\right.$ hours, $20^{\circ} \mathrm{C}$, on plates with food). (b,c) Percent of L4-stage animals to survive hypoxia-starvation $\left(0.5 \% \mathrm{O}_{2}, 48\right.$ hours, $20^{\circ} \mathrm{C}$, in liquid without food). Animals previously grown on plates supplemented with $50 \mathrm{mM}$ PEP are indicated by red bars. ${ }^{* * * *} p<0.0001,{ }^{* *} p<0.01,{ }^{*} p<0.05$ ANOVA/Dunnett's multiple

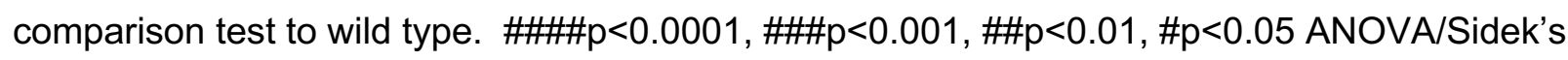
multiple comparison test for the indicated comparisons. (d) Model illustrating how HIF-1 binds near the pck-1 promoter to upregulate PCK-1 expression and drive gluconeogenesis and the production of antioxidants. 


\section{EXTENDED FIGURE LEGENDS}

\section{Extended Data Figure 1. The odls131[hif-1::gfp] Transgene Behaves Like Endogenous} hif-1.

Diagrams of the (a) hif-1 locus and the (b) odls131[hif-1::gfp] transgene. Arrows indicate the TSS. Boxes indicate exons. The black bar indicates sequences removed in the hif-1(ia4) deletion. The red bar indicates the amplicon for the qRT-PCR in (c). The blue and green bars indicate the sequence targets to which RNA-seq reads were aligned in (d) and (e), respectively. (c-e) Relative hif-1 mRNA expression levels determined by (c) qRT-PCR or (d,e) RNA-seq read counts per total million reads in the indicated genetic background. Primers in (c) anneal to sequences inside the hif-1(ia4) deletion and thus only detect transgenic hif-1 transcripts in the egl-9(sa307); hif-1(ia4); odls131[hif-1::gfp] background. RNA-seq sequencing reads were aligned to (d) exons 5-9 outside of the deletion (thus measuring both wild-type transcripts and transcripts containing the deletion), or (e) exons 2-3 inside of the deletion (thus measuring only wild-type transcripts). The homozygous odls131[hif-1::gfp] transgene inserted on the X chromosome; its expression level being about half that of endogenous hif-1 is consistent with the transgene undergoing $\mathrm{X}$-linked dosage compensation. All expression data is normalized to wild type in each graph. $(\mathrm{f}, \mathrm{g})$ Relative mRNA expression levels for the indicated HIF-1 target genes in the indicated genotypes determined by qRT-PCR. (h) Average number of unlaid eggs in utero per animal for the indicated genotype ( $n=40-55$ animals). (i) Average percent of animals alive on the given day of exposure to hypoxia without food ( $n=5$ trials, 50-120 animals per trial). Results in (d-g) demonstrate that the odls131[hif-1::gfp] transgene is sufficient to conduct HIF-1 physiological functions when expressed at the level of endogenous hif-1, which is expected given that all reported hif-1 mutant phenotypes are recessive. For all graphs, error bars indicate SEM. ${ }^{* * *} p<0.0001,{ }^{* * *} p<0.001,{ }^{* *} p<0.01,{ }^{*} \mathrm{P}<0.05$ compared to wild type using ANOVA/Dunnett's multiple comparison test.

\section{Extended Data Figure 2.}

$(a, b)$ Histogram of the number of HIF-1 binding sites (ChIP-seq peaks) against the distance of those peaks from (a) the TSS of the nearest gene, or (b) the stop codon of the nearest gene. Separate graphs are given for the $0-16 \mathrm{~kb}$ or $0-2 \mathrm{~kb}$ range. Most peaks tend to fall within about 500 bps of either the TSS or the stop codon of a nearby gene. (c) Consensus HRE sequences identified by MEME-Suite in humans and enriched in all $C$. elegans ChIP-seq sequences. 


\section{Extended Data Figure 3. RNA-seq of Several Genotypes Identifies High-confidence} Targets.

(a) The four different pairwise comparisons of RNA-seq expression data used to identify genes whose expression is changed when HIF-1 is active versus inactive under aerobic conditions. (b) Multidimensional Scaling analysis of RNA-seq gene expression data for four replicates each of four different genotypes. (c-e) Venn diagrams of the number (and percentage of total) of genes with significant differential expression in the indicated pairwise comparison. (c) Genes that are upregulated when HIF-1 is active. (d) Genes with downregulated expression when HIF1 is active. (e) Direct target genes (i.e., genes with a HIF-1 binding site within $15 \mathrm{~kb}$ ) that are upregulated when HIF-1 is active. No direct target genes are downregulated when HIF-1 is active. (f) The genes egl- 9 and $r h y-1$, which are negative regulators of HIF-1, are some of the strongest upregulated targets of HIF-1, highlighting the importance of negative feedback regulation in the pathway.

\section{Extended Data Figure 4.}

(a) Cartoon of the rhy-1 locus and the different Venus-based promoters used to examine its regulated expression. Boxes indicate exons. Arrows indicate the TSS. The ovals and black lines beneath them indicate HIF-1 and the HIF-1 binding site identified by ChIP-seq, respectively. The inverted triangles indicate the sites of the HRE motif. The yellow arrow indicates sequences encoding the fluorescent Venus reporter. The oac-54 gene is nearby this cluster of HIF-1 binding sites and also shows HIF-1-dependent regulation. (b) Graph of Venus/mCherry fluorescence ratios for the indicated genotype. Bar color indicates specific reporter as per panel A. Error bars indicate SEM. ${ }^{* * *} \mathrm{p}<0.001$ ANOVA/Dunnett's Multiple Comparison test compared to wild type. \#p<0.001 ANOVA/Dunnett's Multiple Comparison test compared to egl-9(sa307). (c) Cumulative probability distribution measuring the number of HIF1 binding sites (ChIP-seq peaks) against the number of other transcription factors known to bind to each site's region of the genome (within 400 bps of each ChIP-seq peak center). The black line indicates all HIF-1 binding sites, whereas the red line indicates HIF-1 binding sites identified by BETA. Blue shading indicates high occupancy target (HOT) sites determined by modENCODE. (d) Graph of the number of times each indicated transcription factor bound within 400 bps of a HIF-1 binding site (ChIP-seq peak center). Specific number is listed above each bar. 


\section{Extended Data Figure 5.}

(a,b) Enriched Gene Ontology (GO) classifications for (a) the 361 genes upregulated when HIF1 is active that are not direct HIF-1 targets, and (b) the 387 genes downregulated when HIF-1 is active. Fold enrichment and FDR for each classification and subclassification is indicated.

\section{Extended Data Figure 6.}

(a,b) Percent of L4-stage animals to survive hypoxia on food $\left(48 \mathrm{~h} 0.1 \% \mathrm{O}_{2}, 25^{\circ} \mathrm{C}\right.$, with $24 \mathrm{~h}$ recovery at $20^{\circ} \mathrm{C}$ ). Because of trial-to-trial variability, survival values for different genotypes within an individual trial are connected by lines. ${ }^{* * *} p<0.001,{ }^{* *} p<0.01,{ }^{*} p<0.05$

ANOVA/Dunnett's multiple comparison test for the average across trials. (c) Average number of unlaid eggs in utero per animal for the indicated genotype ( $n=40-55$ animals). ${ }^{* * *} p<0.0001$, ${ }^{* * *} p<0.001$, ANOVA/Dunnett's multiple comparison test to wild type control. (d) Kaplan-Meier survival curves for animals of the indicated age and for the indicated genotypes. P-values and adjusted FDR values as indicated using the Log-rank test. 


\section{REFERENCES}

1 Vander Heiden, M. G., Cantley, L. C. \& Thompson, C. B. Understanding the Warburg effect: the metabolic requirements of cell proliferation. Science (New York, N.Y 324, 1029-1033, doi:10.1126/science.1160809 (2009).

2 Fandrey, J., Schodel, J., Eckardt, K. U., Katschinski, D. M. \& Wenger, R. H. Now a Nobel gas: oxygen. Pflugers Arch 471, 1343-1358, doi:10.1007/s00424-019-02334-8 (2019).

3 Wang, Z. \& Dong, C. Gluconeogenesis in Cancer: Function and Regulation of PEPCK, FBPase, and G6Pase. Trends Cancer 5, 30-45, doi:10.1016/j.trecan.2018.11.003 (2019).

4 Lopez-Barneo, J. et al. First aid kit for hypoxic survival: sensors and strategies. Physiol Biochem Zool 83, 753-763, doi:10.1086/651584 (2010).

5 Semenza, G. L. et al. Hypoxia, HIF-1, and the pathophysiology of common human diseases. Adv Exp Med Biol 475, 123-130, doi:10.1007/0-306-46825-5_12 (2000).

6 Pullamsetti, S. S., Mamazhakypov, A., Weissmann, N., Seeger, W. \& Savai, R. Hypoxiainducible factor signaling in pulmonary hypertension. J Clin Invest 130, 5638-5651, doi:10.1172/JCl137558 (2020).

7 Wang, B. et al. Effect of hypoxia-inducible factor-prolyl hydroxylase inhibitors on anemia in patients with CKD: a meta-analysis of randomized controlled trials including 2804 patients. Ren Fail 42, 912-925, doi:10.1080/0886022X.2020.1811121 (2020).

8 LaGory, E. L. \& Giaccia, A. J. The ever-expanding role of HIF in tumour and stromal biology. Nature cell biology 18, 356-365, doi:10.1038/ncb3330 (2016).

9 Luo, W. \& Wang, Y. Hypoxia Mediates Tumor Malignancy and Therapy Resistance. Adv Exp Med Biol 1136, 1-18, doi:10.1007/978-3-030-12734-3_1(2019).

10 Zuo, R. J. et al. Warburg-like Glycolysis and Lactate Shuttle in Mouse Decidua during Early Pregnancy. The Journal of biological chemistry 290, 21280-21291, doi:10.1074/jbc.M115.656629 (2015).

11 Faubert, B. et al. AMPK is a negative regulator of the Warburg effect and suppresses tumor growth in vivo. Cell Metab 17, 113-124, doi:10.1016/j.cmet.2012.12.001 (2013).

12 Fernandez-Ramos, A. A., Poindessous, V., Marchetti-Laurent, C., Pallet, N. \& Loriot, M. A. The effect of immunosuppressive molecules on T-cell metabolic reprogramming. Biochimie 127, 23-36, doi:10.1016/j.biochi.2016.04.016 (2016).

13 Kim, J. W., Tchernyshyov, I., Semenza, G. L. \& Dang, C. V. HIF-1-mediated expression of pyruvate dehydrogenase kinase: a metabolic switch required for cellular adaptation to hypoxia. Cell Metab 3, 177-185, doi:10.1016/j.cmet.2006.02.002 (2006).

14 Papandreou, I., Cairns, R. A., Fontana, L., Lim, A. L. \& Denko, N. C. HIF-1 mediates adaptation to hypoxia by actively downregulating mitochondrial oxygen consumption. Cell Metab 3, 187-197, doi:10.1016/j.cmet.2006.01.012 (2006).

15 Seagroves, T. N. et al. Transcription factor HIF-1 is a necessary mediator of the pasteur effect in mammalian cells. Molecular and cellular biology 21, 3436-3444, doi:10.1128/MCB.21.10.3436-3444.2001 (2001).

16 Iyer, N. V. et al. Cellular and developmental control of $\mathrm{O} 2$ homeostasis by hypoxiainducible factor 1 alpha. Genes Dev 12, 149-162, doi:10.1101/gad.12.2.149 (1998).

17 Semenza, G. L., Roth, P. H., Fang, H. M. \& Wang, G. L. Transcriptional regulation of genes encoding glycolytic enzymes by hypoxia-inducible factor 1 . The Journal of biological chemistry 269, 23757-23763 (1994).

18 Schodel, J. et al. High-resolution genome-wide mapping of HIF-binding sites by ChIPseq. Blood 117, e207-217, doi:10.1182/blood-2010-10-314427 (2011). 
19 Benita, Y. et al. An integrative genomics approach identifies Hypoxia Inducible Factor-1 (HIF-1)-target genes that form the core response to hypoxia. Nucleic Acids Res 37, 4587-4602, doi:10.1093/nar/gkp425 (2009).

20 de Bruin, A. et al. Genome-wide analysis reveals NRP1 as a direct HIF1alpha-E2F7 target in the regulation of motorneuron guidance in vivo. Nucleic Acids Res 44, 35493566, doi:10.1093/nar/gkv1471 (2016).

21 Mimura, I. et al. Dynamic change of chromatin conformation in response to hypoxia enhances the expression of GLUT3 (SLC2A3) by cooperative interaction of hypoxiainducible factor 1 and KDM3A. Molecular and cellular biology 32, 3018-3032, doi:10.1128/MCB.06643-11 (2012).

22 Anderson, G. L. \& Dusenbery, D. B. Critical-Oxygen Tension of Caenorhabdiltis elegans. J Nematol 9, 253-254 (1977).

23 Powell-Coffman, J. A. Hypoxia signaling and resistance in C. elegans. Trends Endocrinol Metab 21, 435-440, doi:10.1016/j.tem.2010.02.006 (2010).

24 Trent, C., Tsuing, N. \& Horvitz, H. R. Egg-laying defective mutants of the nematode Caenorhabditis elegans. Genetics 104, 619-647 (1983).

25 Shen, C., Shao, Z. \& Powell-Coffman, J. A. The Caenorhabditis elegans rhy-1 gene inhibits HIF-1 hypoxia-inducible factor activity in a negative feedback loop that does not include vhl-1. Genetics 174, 1205-1214 (2006).

26 Shen, C., Nettleton, D., Jiang, M., Kim, S. K. \& Powell-Coffman, J. A. Roles of the HIF-1 hypoxia-inducible factor during hypoxia response in Caenorhabditis elegans. The Journal of biological chemistry 280, 20580-20588 (2005).

27 Chang, A. J. \& Bargmann, C. I. Hypoxia and the HIF-1 transcriptional pathway reorganize a neuronal circuit for oxygen-dependent behavior in Caenorhabditis elegans. Proceedings of the National Academy of Sciences of the United States of America 105, 7321-7326 (2008).

28 Pocock, R. \& Hobert, O. Oxygen levels affect axon guidance and neuronal migration in Caenorhabditis elegans. Nature neuroscience 11, 894-900 (2008).

29 Miller, D. L. \& Roth, M. B. C. elegans are protected from lethal hypoxia by an embryonic diapause. Current biology : CB 19, 1233-1237, doi:10.1016/j.cub.2009.05.066 (2009).

30 Hasse, S., Hyman, A. A. \& Sarov, M. TransgeneOmics--A transgenic platform for protein localization based function exploration. Methods 96, 69-74, doi:10.1016/j.ymeth.2015.10.005 (2016).

31 Ma, D. K., Vozdek, R., Bhatla, N. \& Horvitz, H. R. CYSL-1 interacts with the O2-sensing hydroxylase EGL-9 to promote H2S-modulated hypoxia-induced behavioral plasticity in C. elegans. Neuron 73, 925-940, doi:10.1016/j.neuron.2011.12.037 (2012).

32 Miyabayashi, T., Palfreyman, M. T., Sluder, A. E., Slack, F. \& Sengupta, P. Expression and function of members of a divergent nuclear receptor family in Caenorhabditis elegans. Dev Biol 215, 314-331, doi:10.1006/dbio.1999.9470 (1999).

33 Bishop, T. et al. Genetic analysis of pathways regulated by the von Hippel-Lindau tumor suppressor in Caenorhabditis elegans. PLoS biology 2, e289 (2004).

34 Semenza, G. L. \& Wang, G. L. A nuclear factor induced by hypoxia via de novo protein synthesis binds to the human erythropoietin gene enhancer at a site required for transcriptional activation. Molecular and cellular biology 12, 5447-5454, doi:10.1128/mcb.12.12.5447 (1992).

35 Levy, A. P., Levy, N. S., Wegner, S. \& Goldberg, M. A. Transcriptional regulation of the rat vascular endothelial growth factor gene by hypoxia. The Journal of biological chemistry 270, 13333-13340, doi:10.1074/jbc.270.22.13333 (1995).

36 Wang, S. et al. Target analysis by integration of transcriptome and ChIP-seq data with BETA. Nature protocols 8, 2502-2515, doi:10.1038/nprot.2013.150 (2013). 
37 Bailey, T. L. et al. MEME SUITE: tools for motif discovery and searching. Nucleic Acids Res 37, W202-208, doi:10.1093/nar/gkp335 (2009).

38 Kudron, M. M. et al. The ModERN Resource: Genome-Wide Binding Profiles for Hundreds of Drosophila and Caenorhabditis elegans Transcription Factors. Genetics 208, 937-949, doi:10.1534/genetics.117.300657 (2018).

39 Wreczycka, K. et al. HOT or not: examining the basis of high-occupancy target regions. Nucleic Acids Res 47, 5735-5745, doi:10.1093/nar/gkz460 (2019).

40 An, J. H. \& Blackwell, T. K. SKN-1 links C. elegans mesendodermal specification to a conserved oxidative stress response. Genes Dev 17, 1882-1893 (2003).

41 Heimbucher, T., Hog, J., Gupta, P. \& Murphy, C. T. PQM-1 controls hypoxic survival via regulation of lipid metabolism. Nature communications 11, 4627, doi:10.1038/s41467020-18369-w (2020).

42 Luhachack, L. G. et al. EGL-9 controls C. elegans host defense specificity through prolyl hydroxylation-dependent and -independent HIF-1 pathways. PLoS pathogens 8 , e1002798, doi:10.1371/journal.ppat.1002798 (2012).

43 Shao, Z., Zhang, Y., Ye, Q., Saldanha, J. N. \& Powell-Coffman, J. A. C. elegans SWAN1 Binds to EGL-9 and regulates HIF-1-mediated resistance to the bacterial pathogen Pseudomonas aeruginosa PAO1. PLoS pathogens 6, doi:10.1371/journal.ppat.1001075 (2010).

44 Budde, M. W. \& Roth, M. B. Hydrogen sulfide increases hypoxia-inducible factor-1 activity independently of von Hippel-Lindau tumor suppressor-1 in C. elegans. Molecular biology of the cell 21, 212-217, doi:10.1091/mbc.E09-03-0199 (2010).

45 Gallagher, L. A. \& Manoil, C. Pseudomonas aeruginosa PAO1 kills Caenorhabditis elegans by cyanide poisoning. J Bacteriol 183, 6207-6214, doi:10.1128/JB.183.21.62076214.2001 (2001).

46 Davis, W. L. \& Goodman, D. B. Evidence for the glyoxylate cycle in human liver. Anat Rec 234, 461-468, doi:10.1002/ar.1092340402 (1992).

47 Strittmatter, L. et al. CLYBL is a polymorphic human enzyme with malate synthase and beta-methylmalate synthase activity. Human molecular genetics 23, 2313-2323, doi:10.1093/hmg/ddt624 (2014).

48 Schweinsberg, P. J. \& Grant, B. D. C. elegans gene transformation by microparticle bombardment. WormBook, 1-10, doi:10.1895/wormbook.1.166.1 (2013).

49 Schindelin, J. et al. Fiji: an open-source platform for biological-image analysis. Nature methods 9, 676-682, doi:10.1038/nmeth.2019 (2012).

50 Senchuk, M. M., Dues, D. J. \& Van Raamsdonk, J. M. Measuring Oxidative Stress in Caenorhabditis elegans: Paraquat and Juglone Sensitivity Assays. Bio Protoc 7, doi:10.21769/BioProtoc.2086 (2017).

51 Chatterjee, I. et al. Dramatic fertility decline in aging C. elegans males is associated with mating execution deficits rather than diminished sperm quality. Experimental gerontology 48, 1156-1166, doi:10.1016/j.exger.2013.07.014 (2013). 
Figure 1. Vora et al.

a

Wild Type

egl-9(sa307)

$\mathrm{O}_{2}$

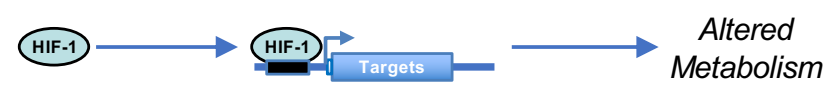

Metabolism

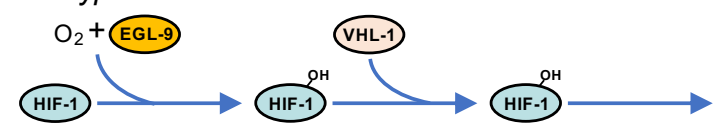

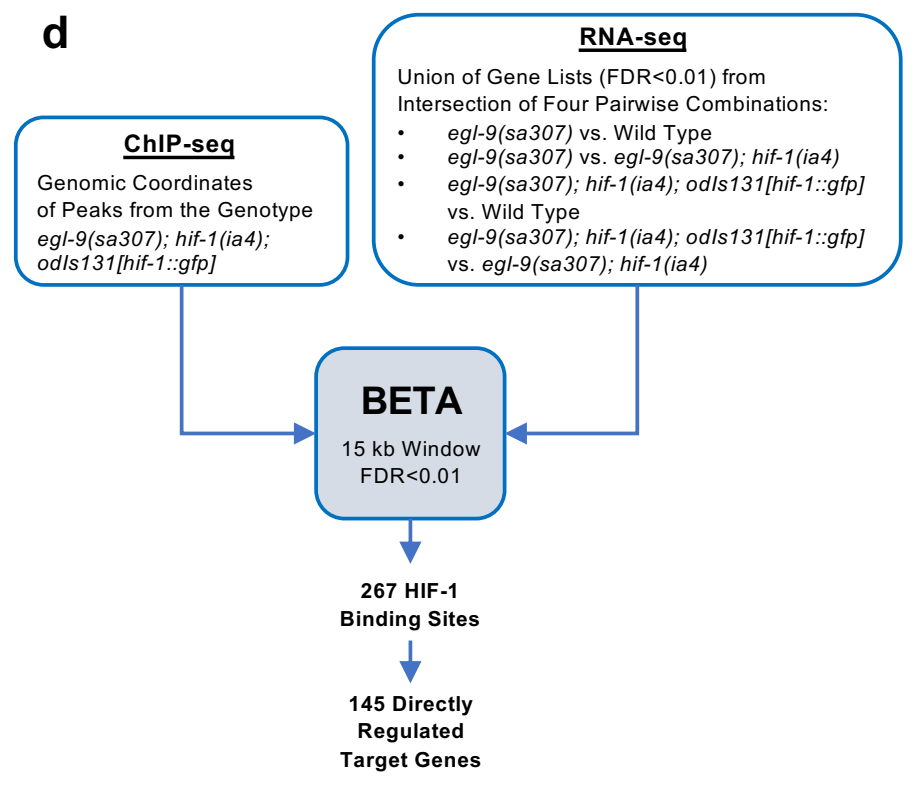

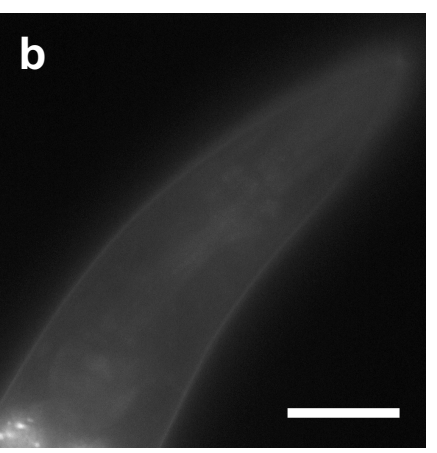

hif-1(ia4); odls131[hif-1::gfp]

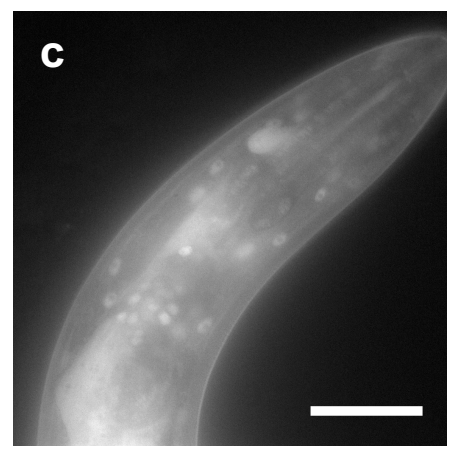

egl-9(sa307); hif-1(ia4); odls131[hif-1::gfp] e

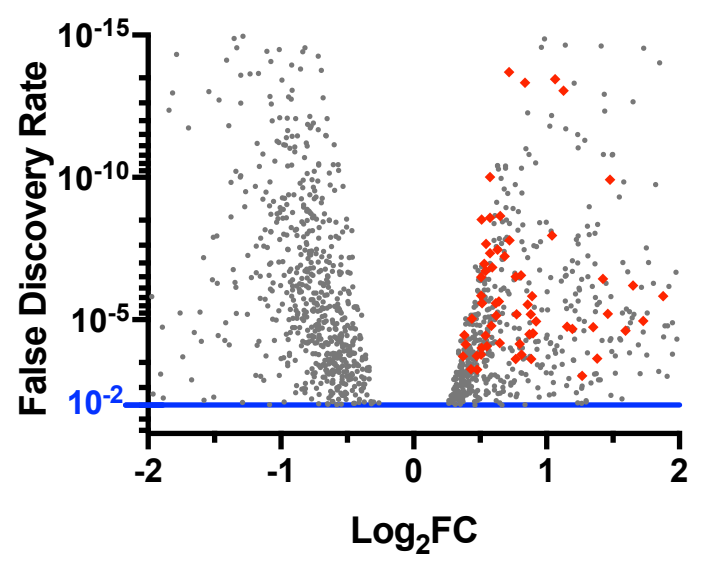

g

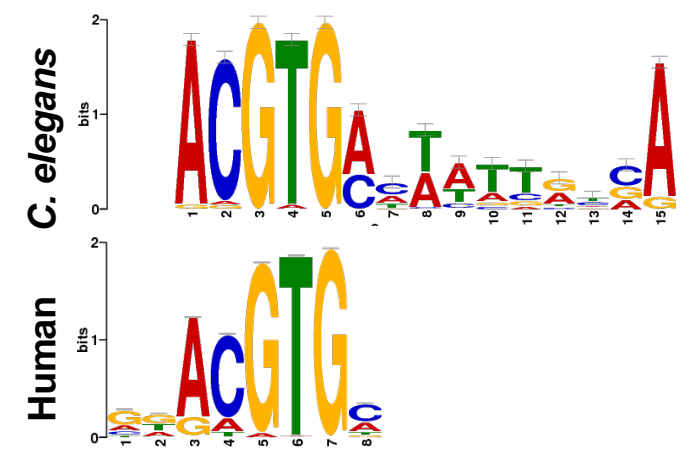

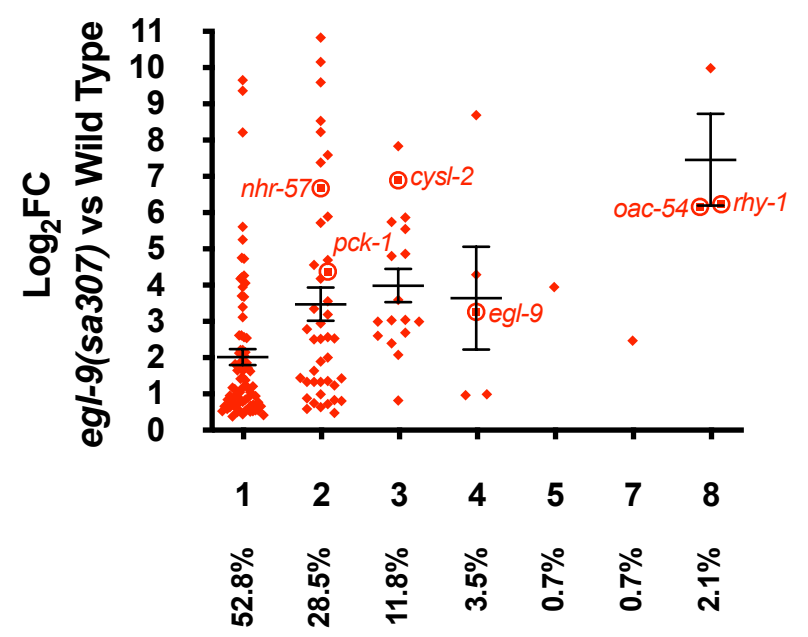

Number of HIF-1 Binding Sites ( $\%$ of Total With That Number) 
Figure 2. Vora et al.

a
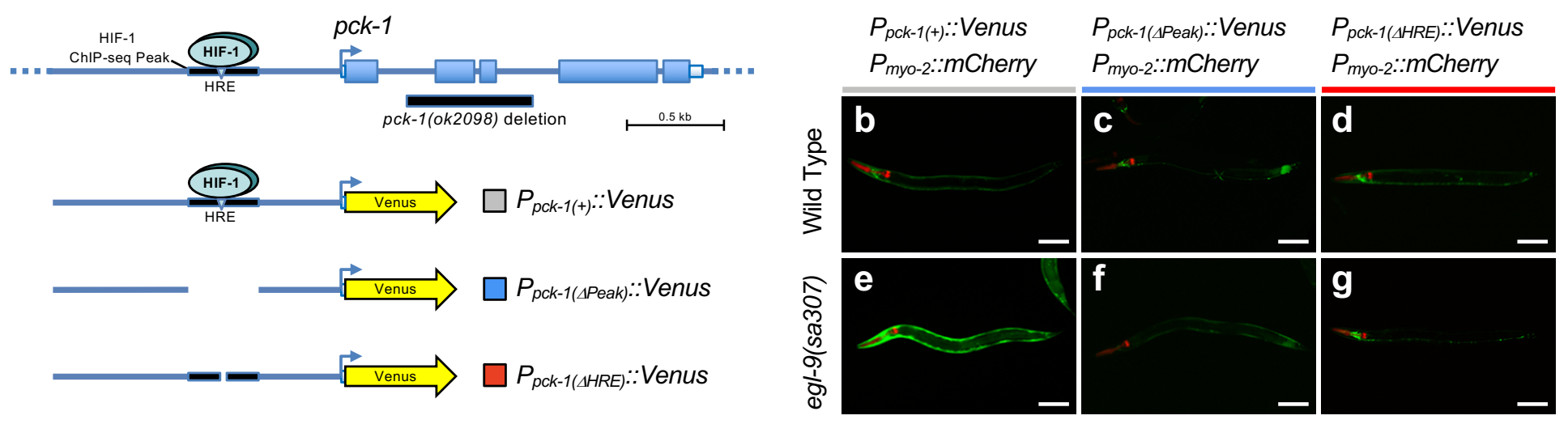

h

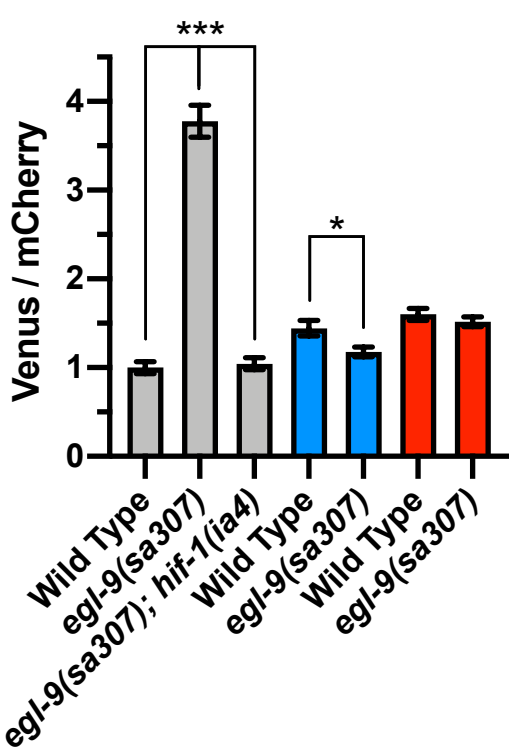

i

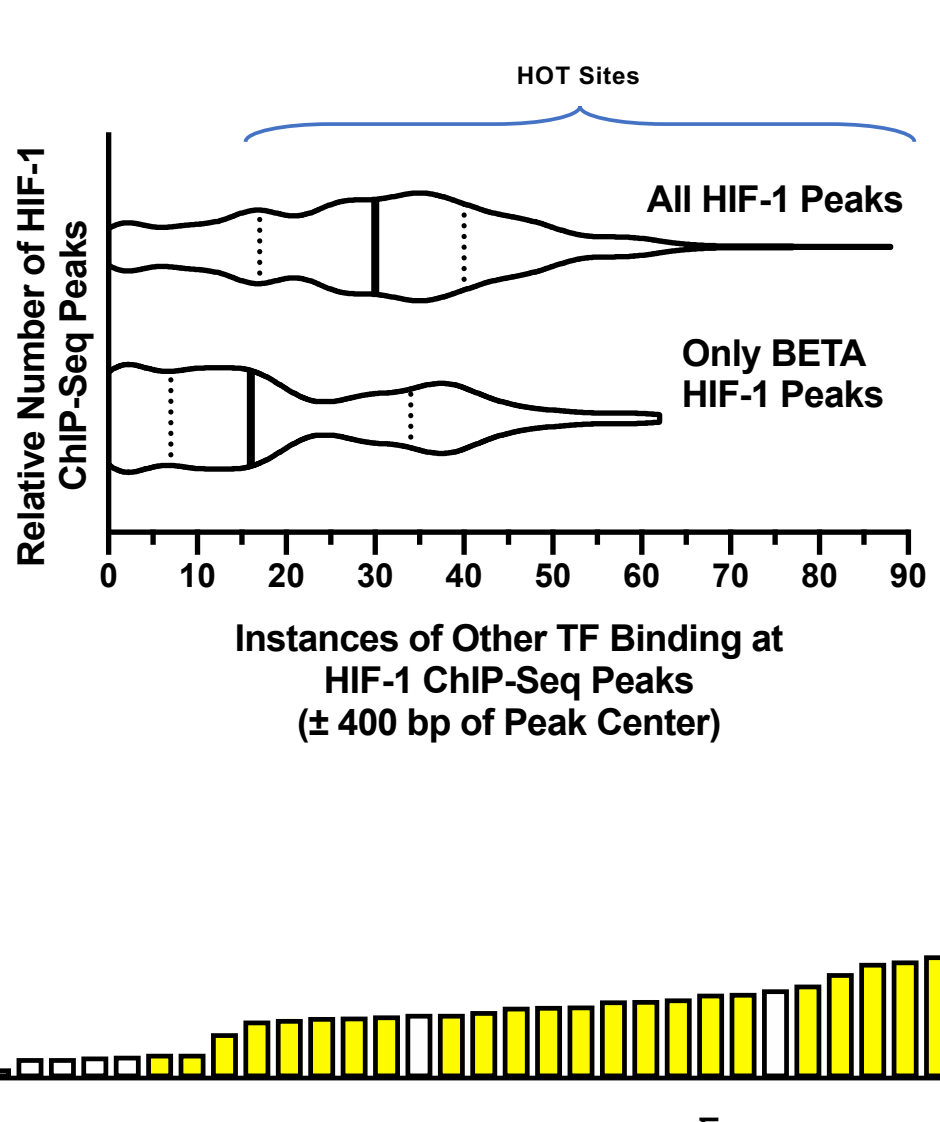

j

究

-

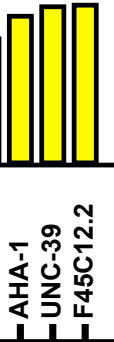

Transcription Factor 
Figure 3. Vora et al.

a

\begin{tabular}{lrrr}
\hline Gene Ontology of $\mathbf{1 4 5}$ Targets Directly Upregulated by HIF-1 & \\
\hline Process & $\begin{array}{r}\text { Number } \\
\text { of Genes }\end{array}$ & $\begin{array}{r}\text { Fold } \\
\text { Enrichment }\end{array}$ & FDR \\
\hline Glucose Metabolism & 22 & 35.8 & $1.1 \times 10^{-7}$ \\
• Glycolysis $\quad$ Gluconeogenesis & 14 & 37.0 & $8.6 \times 10^{-5}$ \\
Amino Acid Metabolism & 14 & 56.3 & $8.2 \times 10^{-9}$ \\
• Serine Biosynthesis & 12 & 8.5 & $7.0 \times 10^{-6}$ \\
• Sulfur/Cysteine Metabolism & 3 & 88.7 & $1.9 \times 10^{-3}$ \\
Fatty Acid Metabolism & 6 & 34.1 & $1.2 \times 10^{-5}$ \\
$\bullet \quad$ Beta-Oxidation & 6 & 7.3 & $2.2 \times 10^{-2}$ \\
Response to O 2 Levels & 4 & 21.9 & $5.7 \times 10^{-3}$ \\
Oxidation-Reduction & 5 & 23.1 & $6.4 \times 10^{-4}$ \\
\hline
\end{tabular}

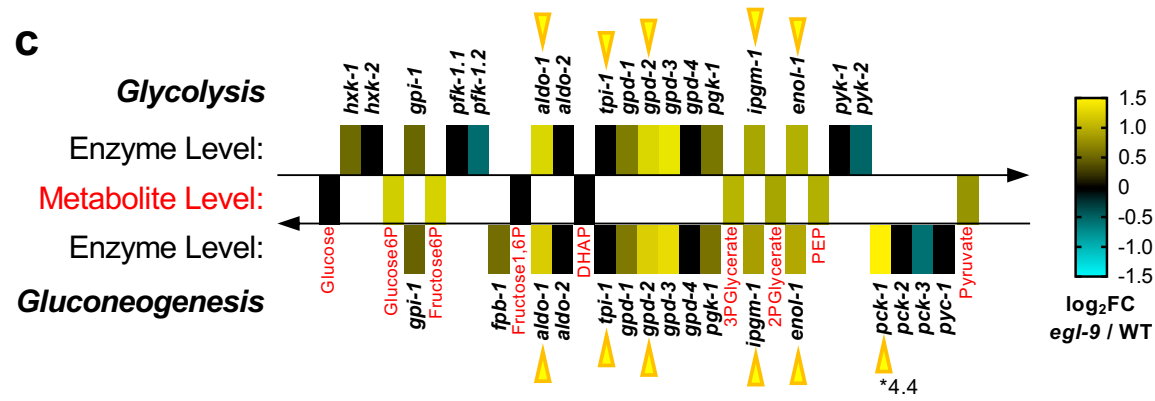

d

Citric Acid Cycle

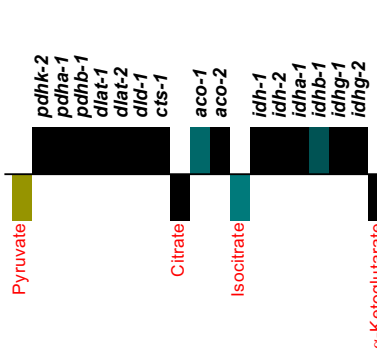

Enzyme Level:

Metabolite Level:

\section{f}
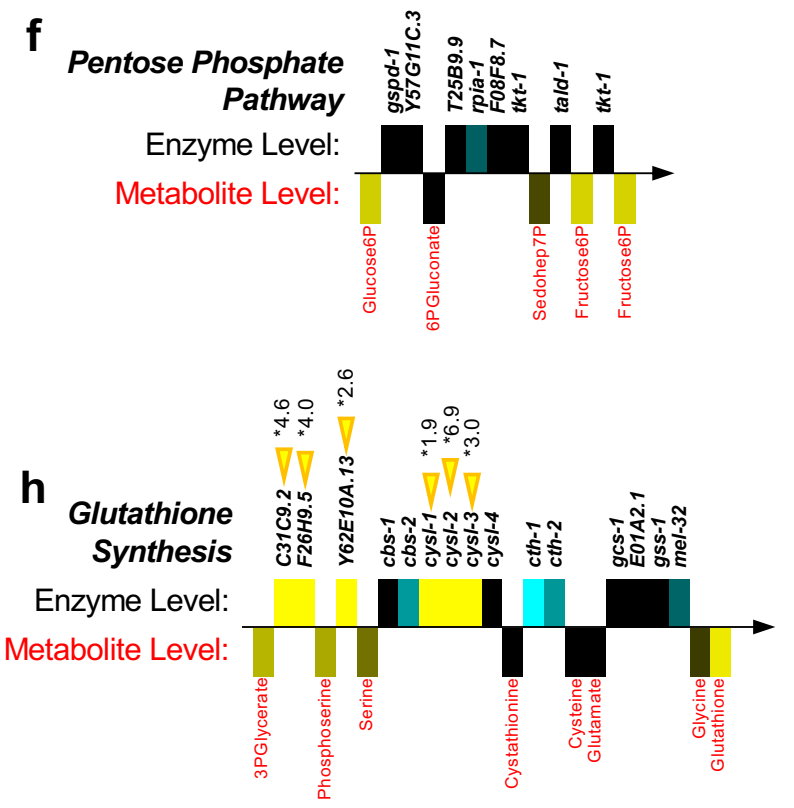

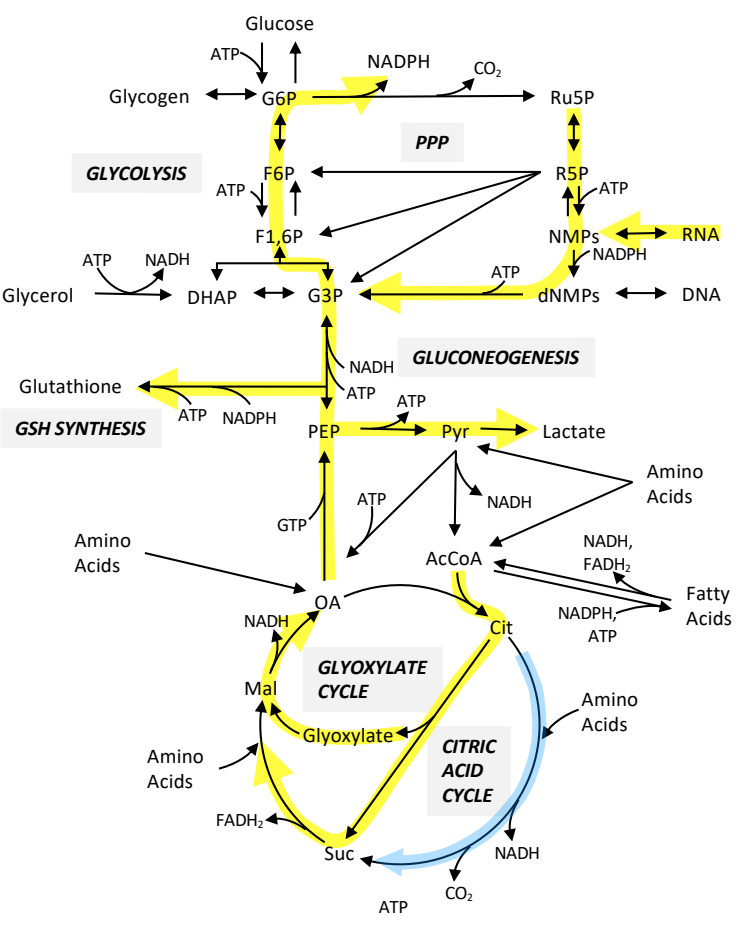

e

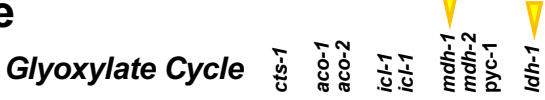

Enzyme Level:

Metabolite Level:

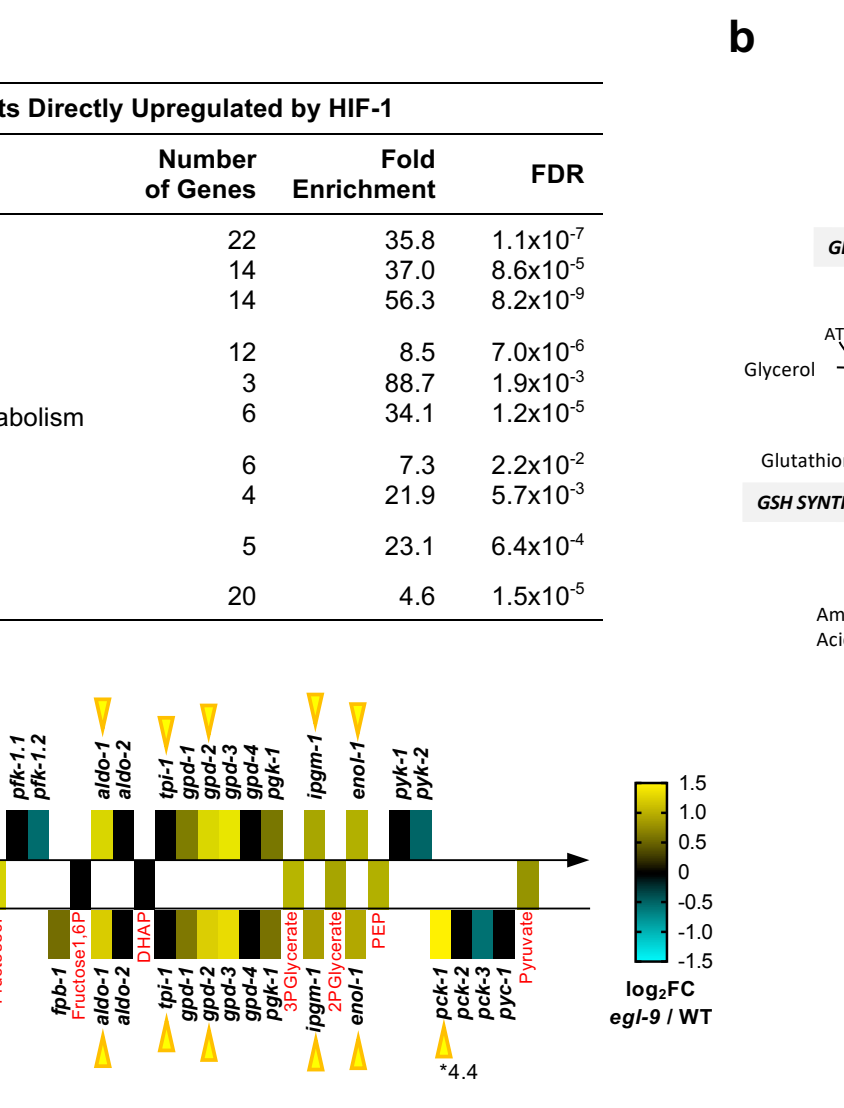
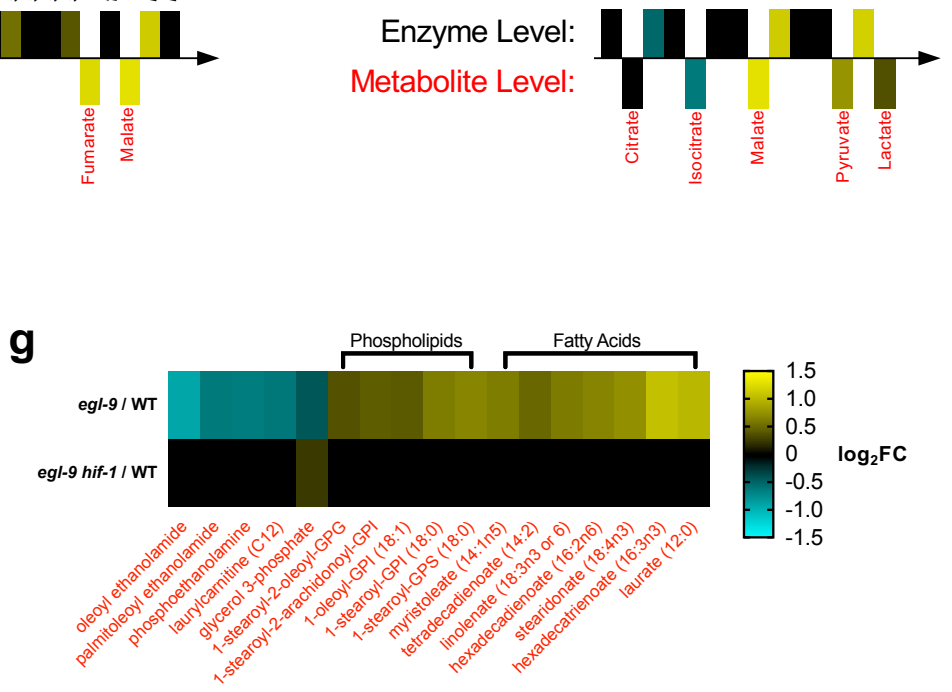

Enzyme Level:

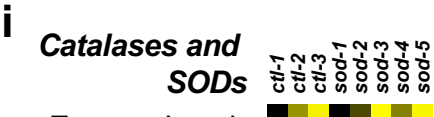

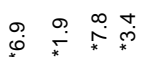

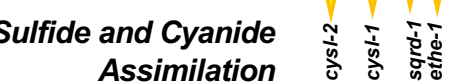

Assimilation

Enzyme Level: 
Figure 4. Vora et al.

a

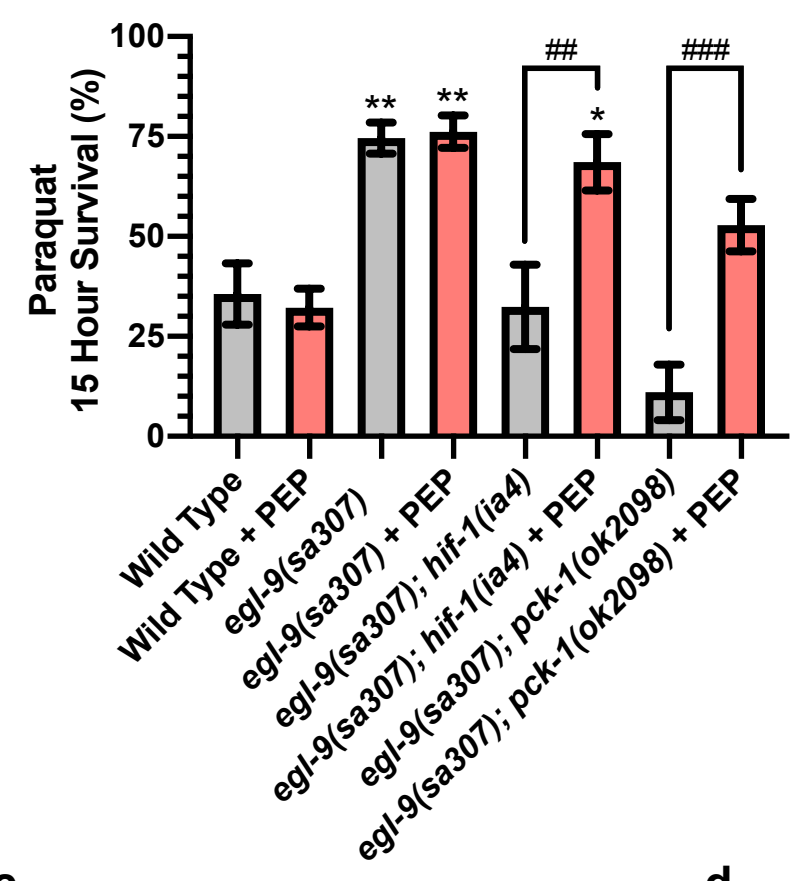

b

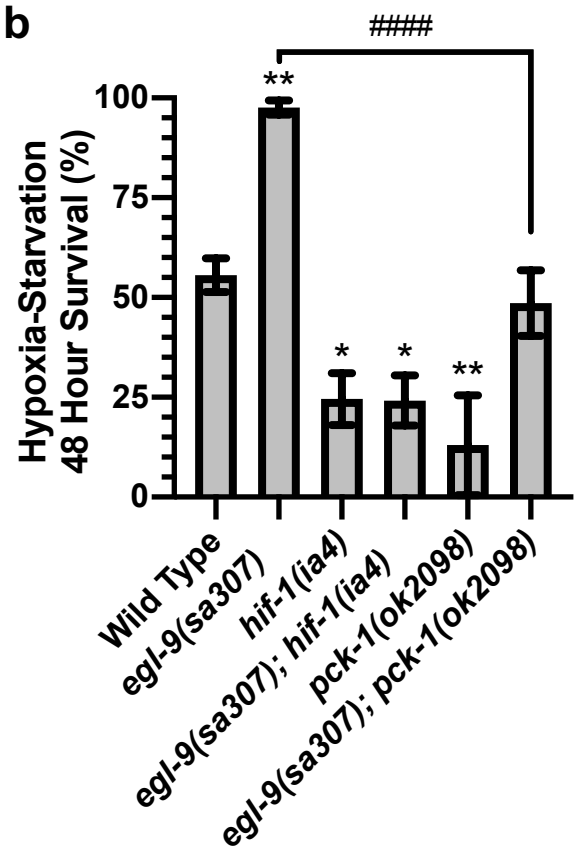

C

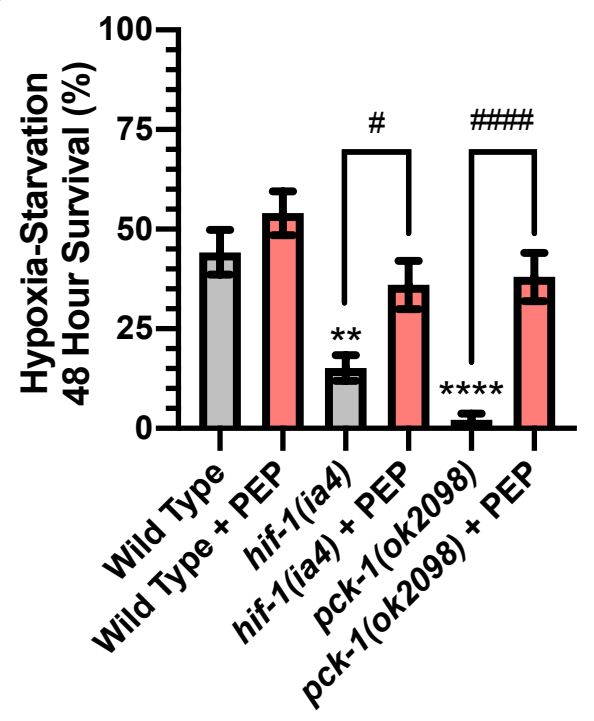

d

HIF-1 Activation Under Aerobic Conditions

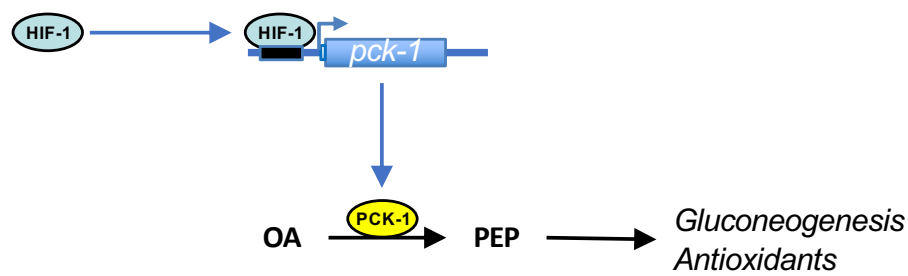


a
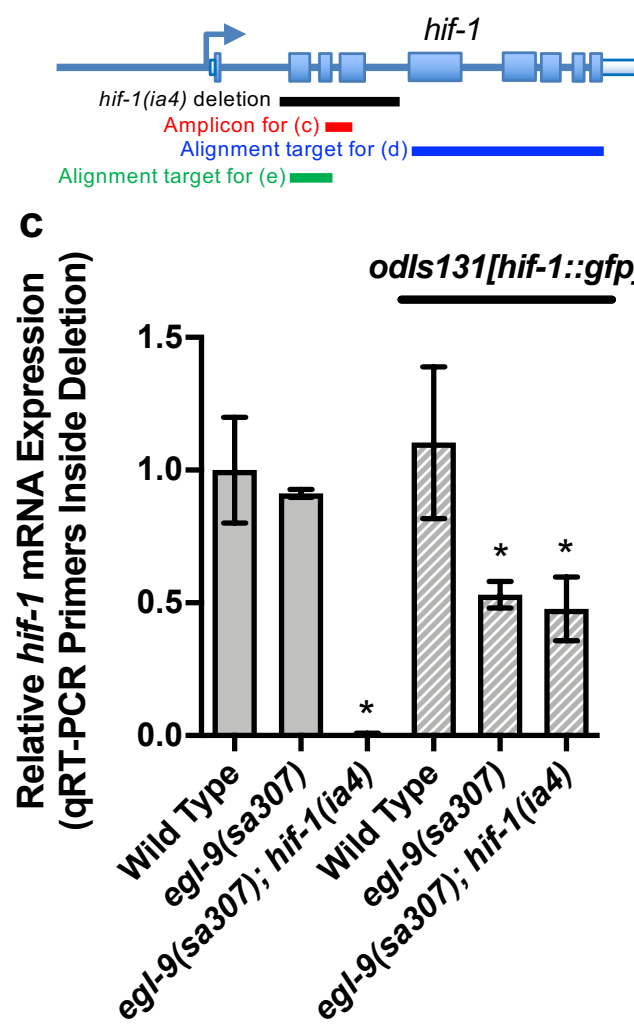

f

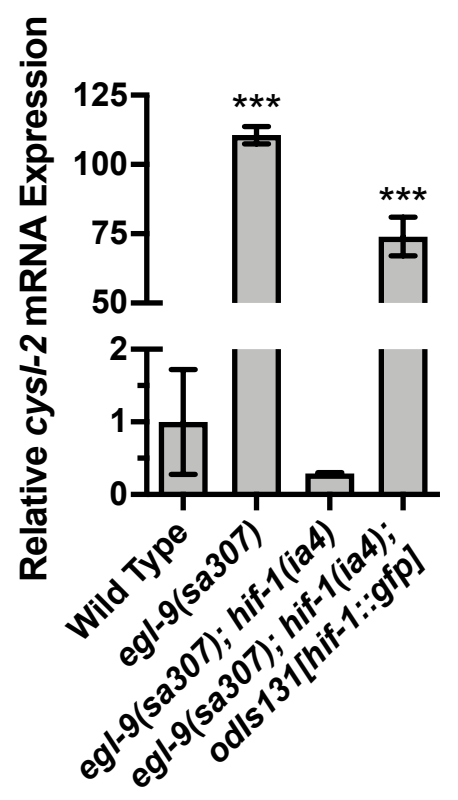

i

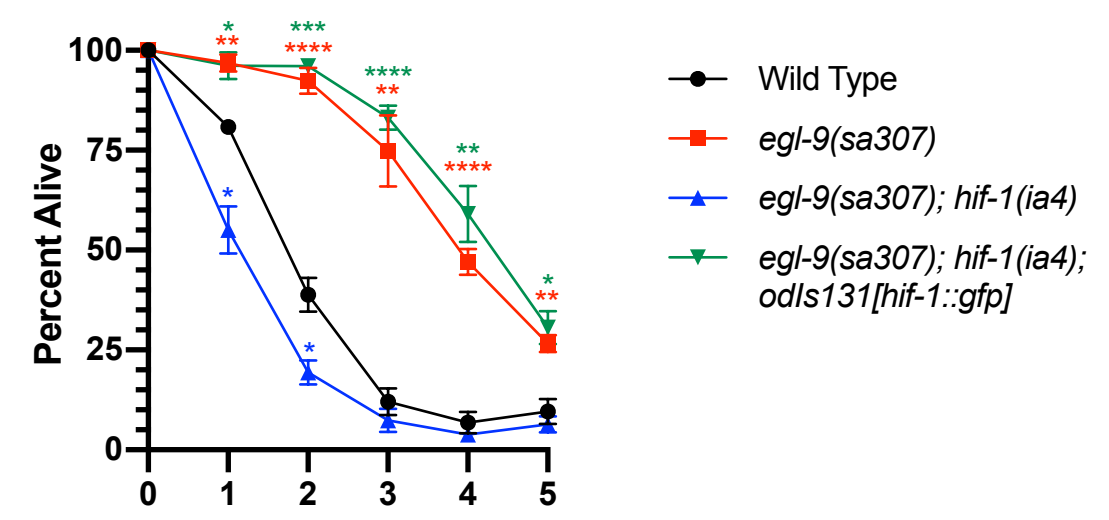

b

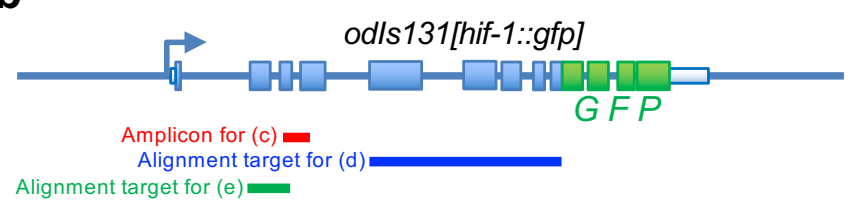

d

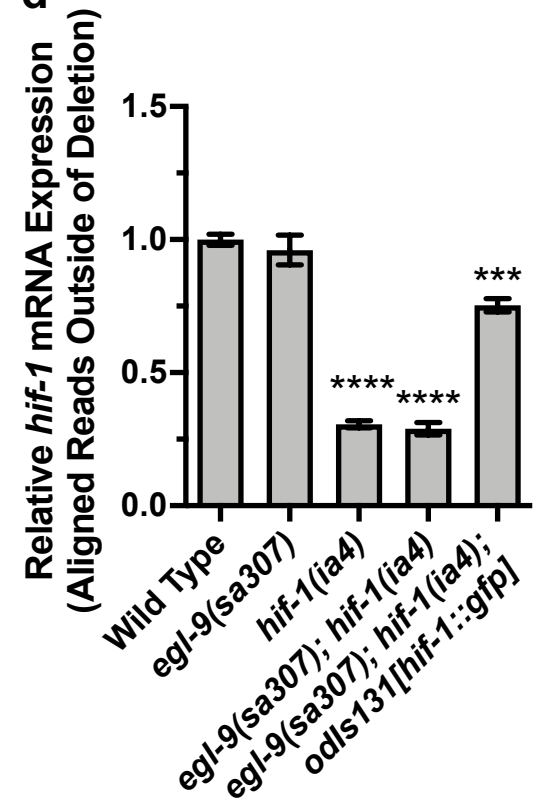

g
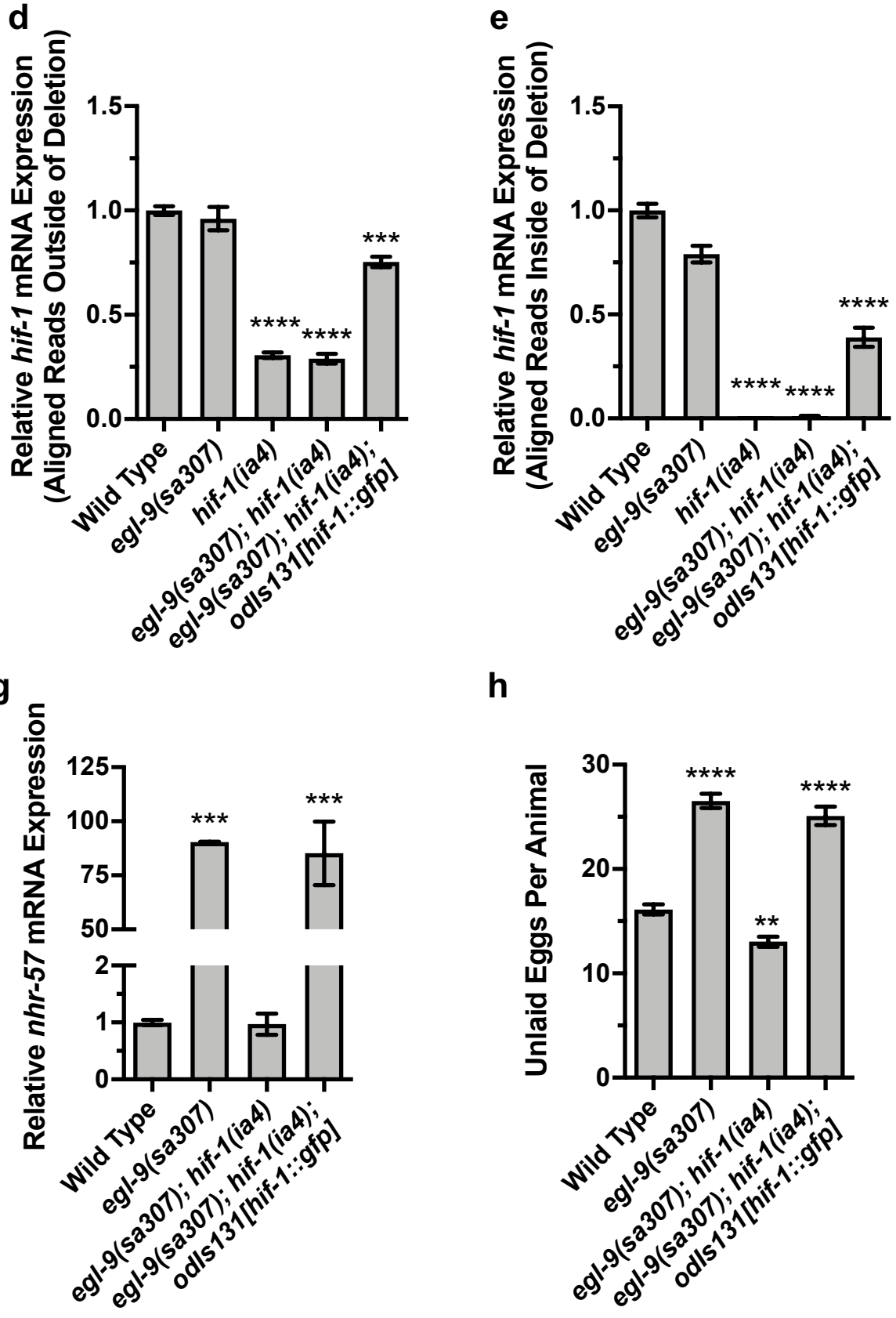

h

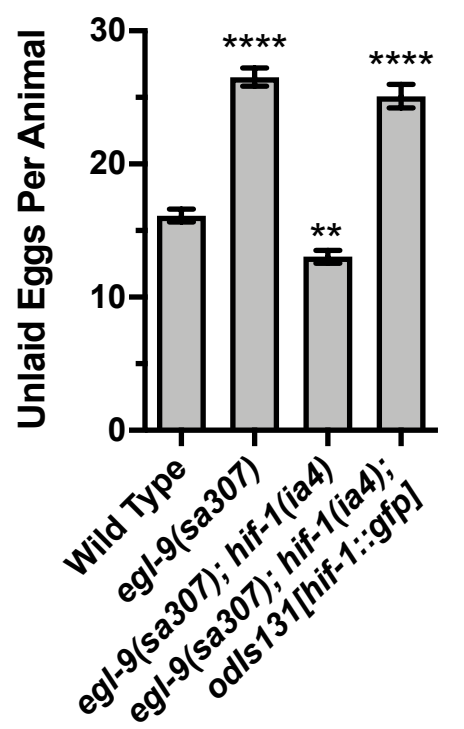




\section{Extended Data Figure 2. Vora et al.}

\section{a Distance of HIF-1 ChIP-seq peak to nearest CDS START} $0-16 \mathrm{~kb}$

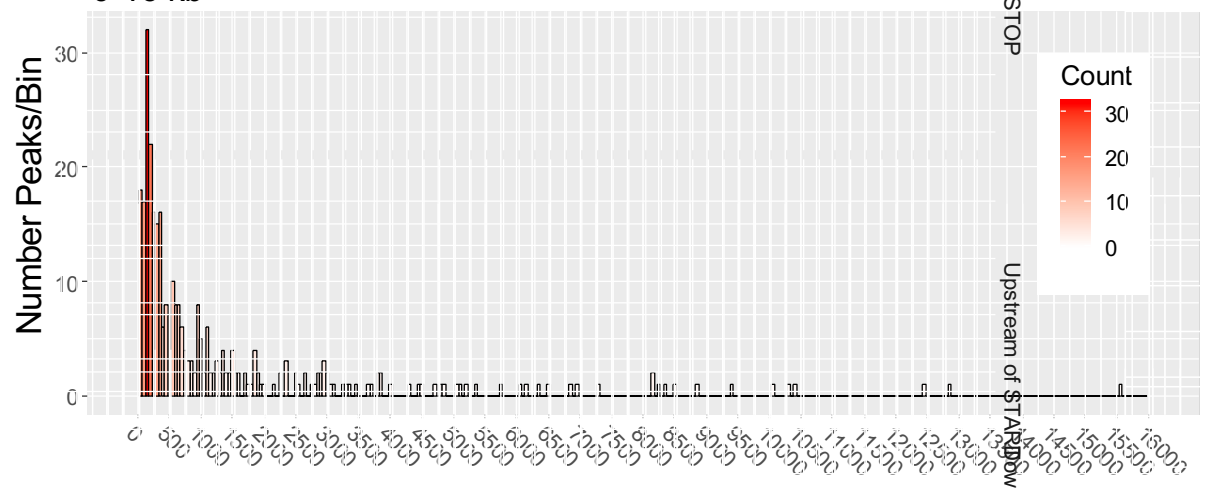

b

\section{Distance of HIF-1 ChIP-seq peak to nearest CDS STOP} $0-16 \mathrm{~kb}$

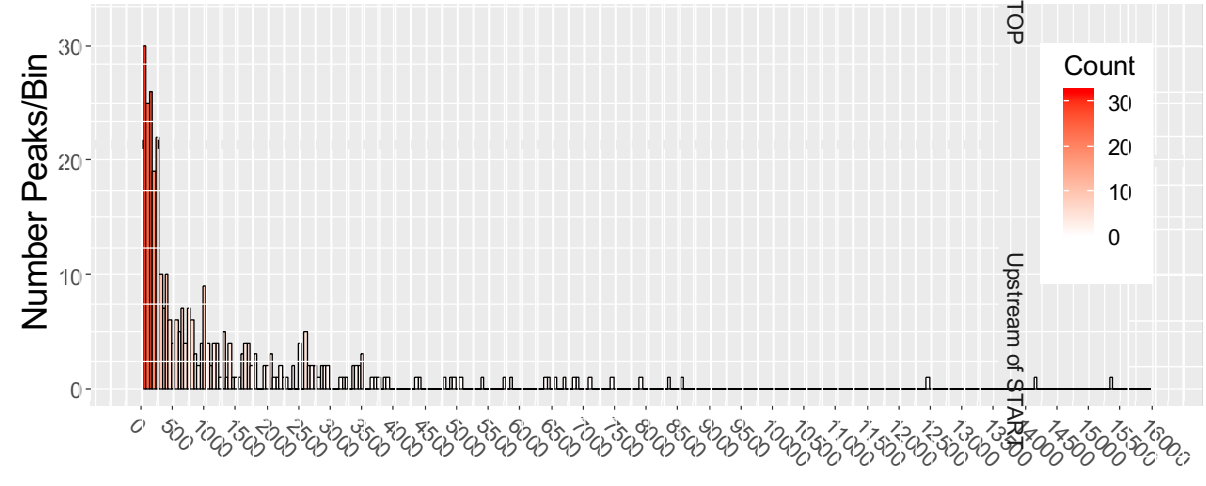

C

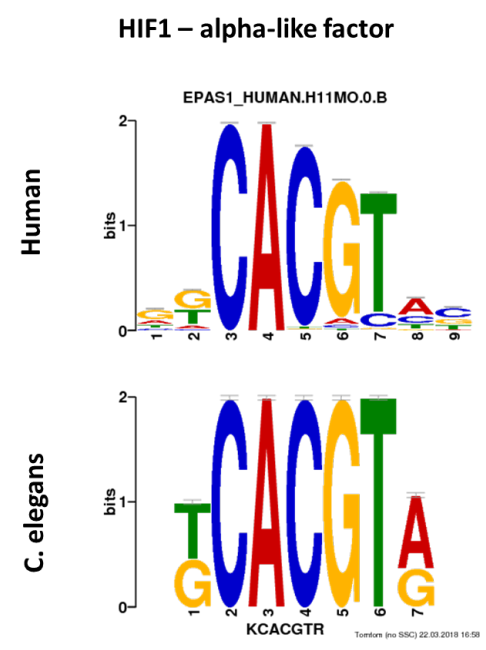

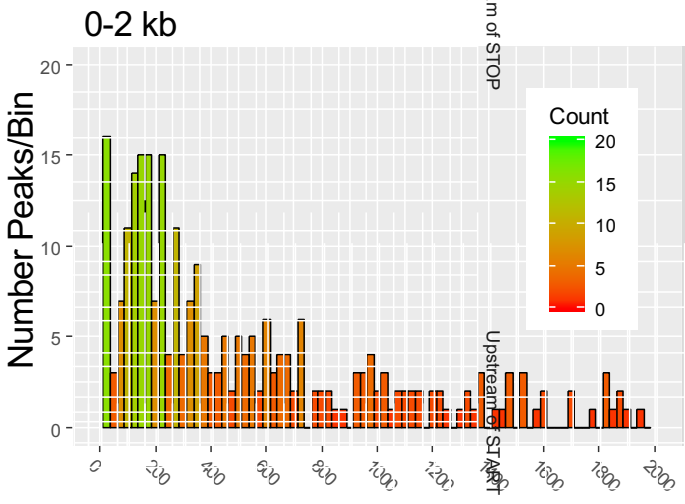

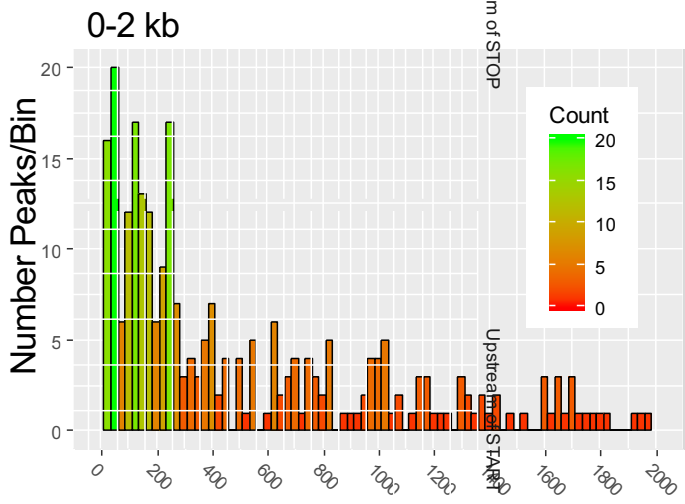




\section{Extended Data Figure 3. Vora et al.}

a

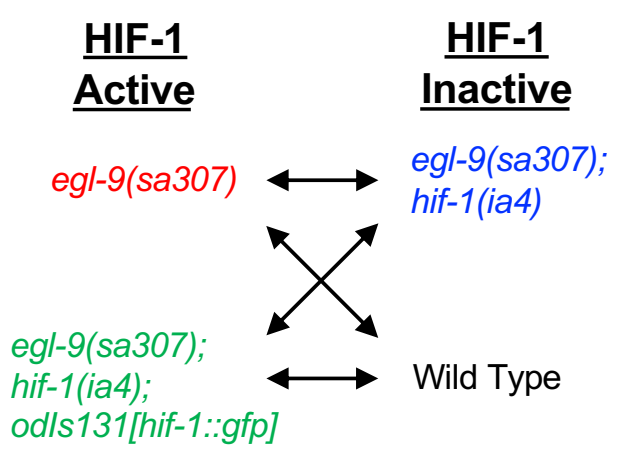

C

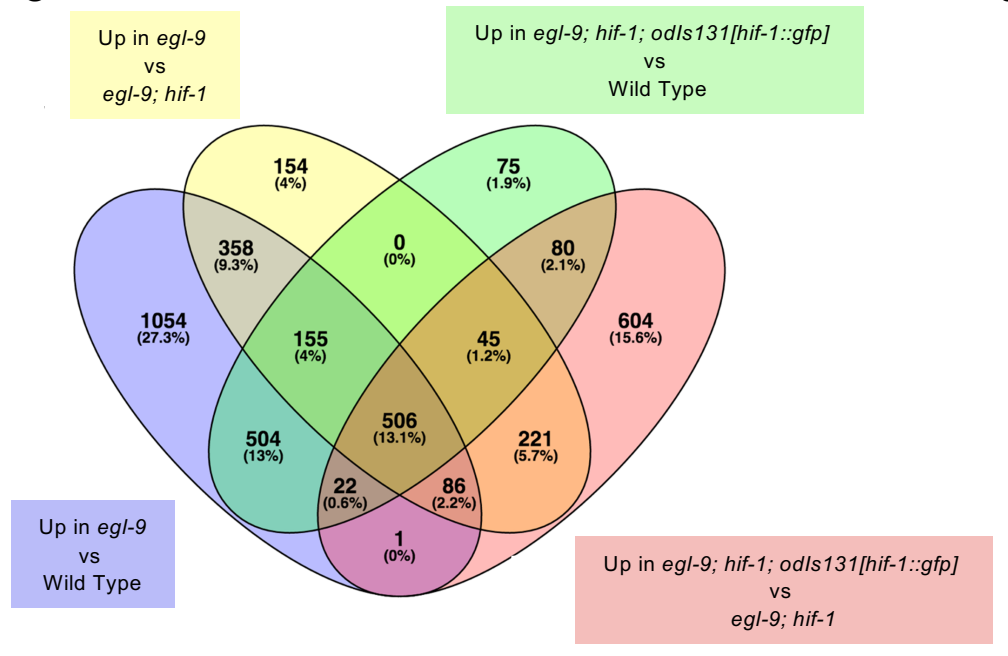

e

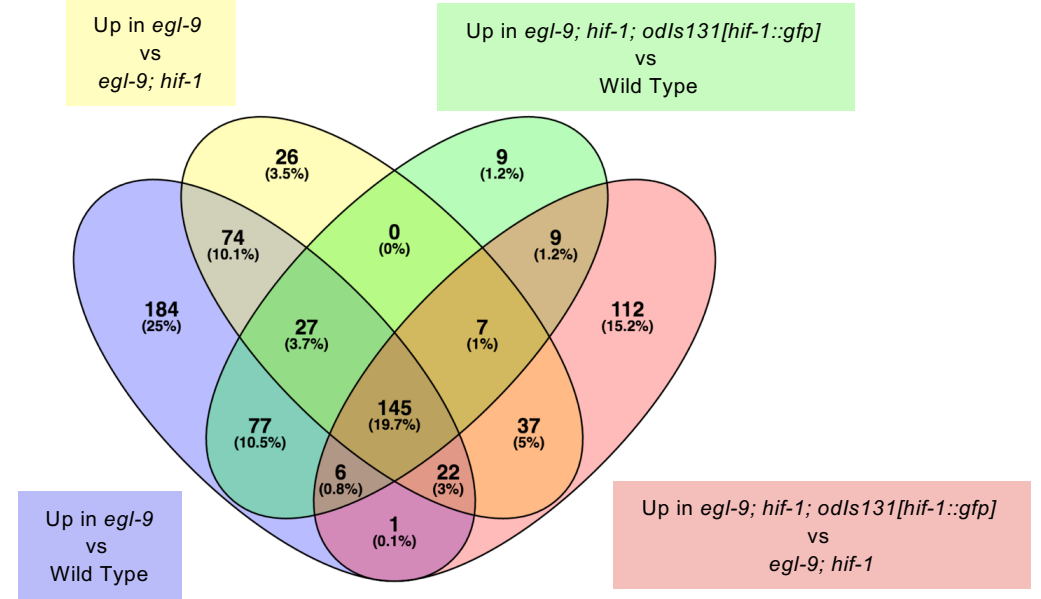

b

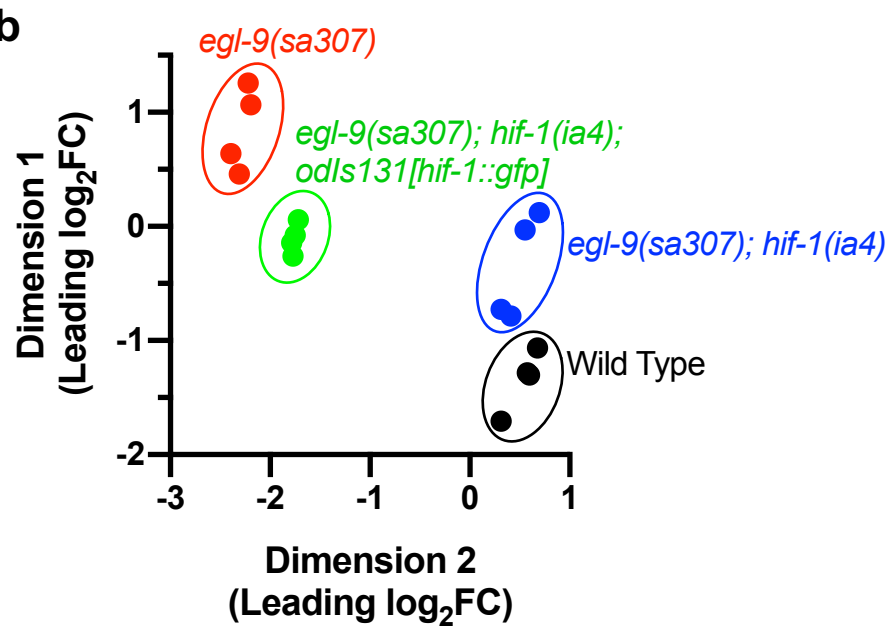

d
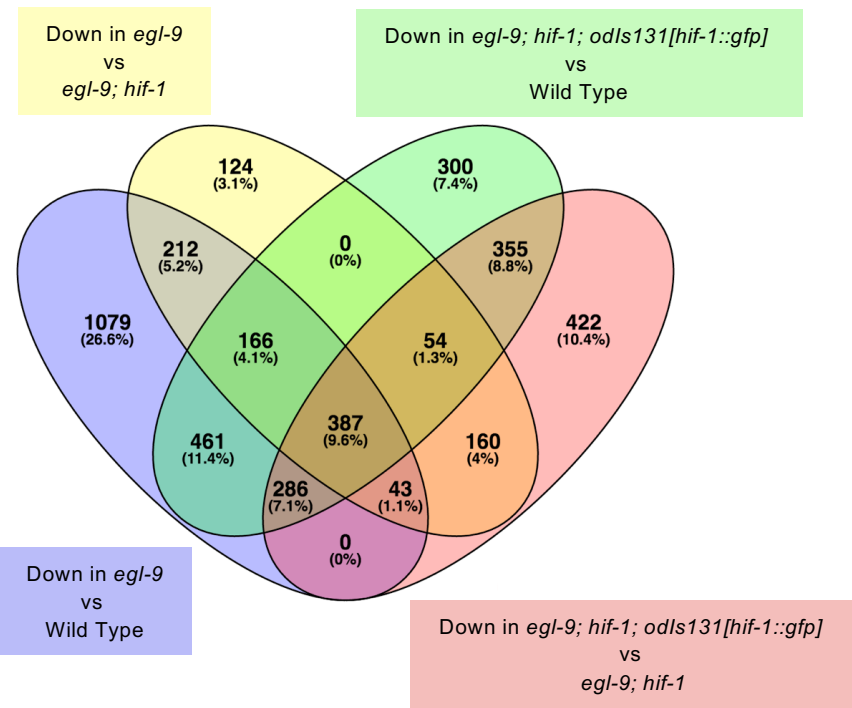

f

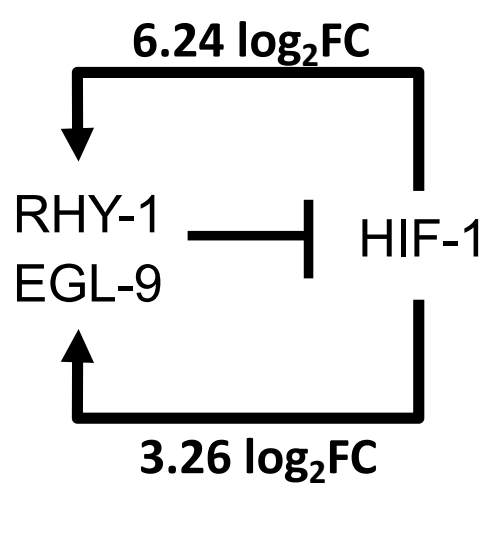




\section{Extended Data Figure 4. Vora et al.}

a
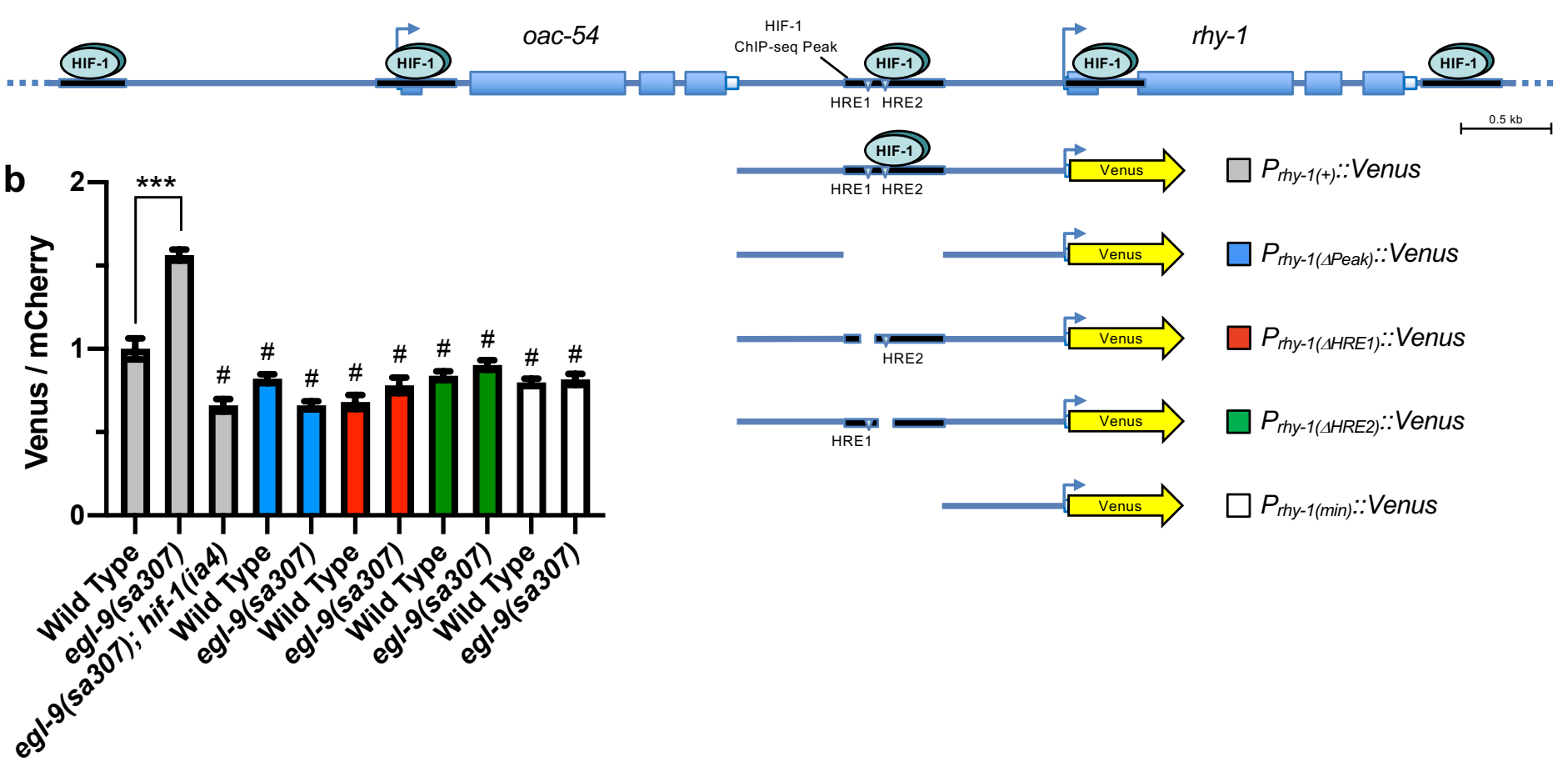

C

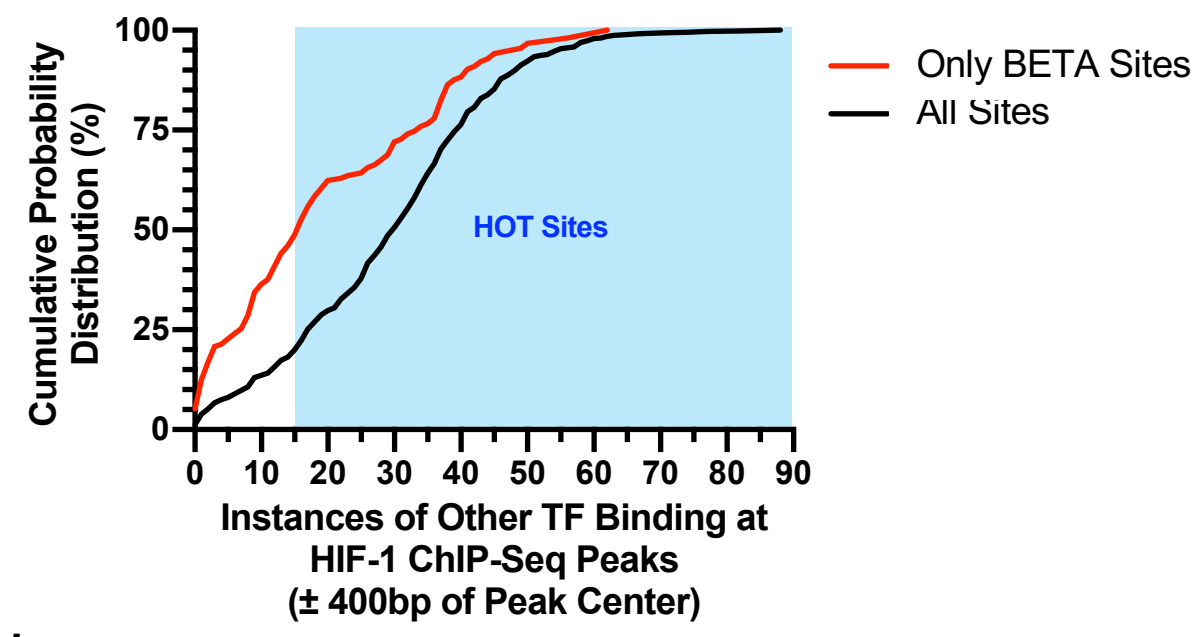

d

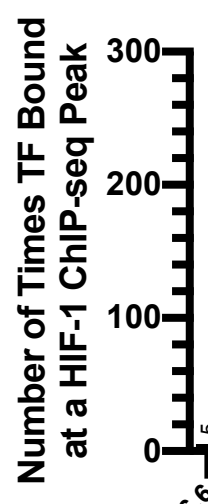


bioRxiv preprint doi: https://doi.org/10.1101/2021.05.04.442650; this version posted May 4, 2021. The copyright holder for this preprint (which was not certified by peer review) is the author/funder. All rights reserved. No reuse allowed without permission.

\section{Extended Data Figure 5. Vora et al.}

a

\begin{tabular}{lrrr}
\hline Gene Ontology of $\mathbf{3 6 1}$ Targets Indirectly Upregulated by HIF-1 & \\
\hline Process & $\begin{array}{r}\text { Number } \\
\text { of Genes }\end{array}$ & $\begin{array}{r}\text { Fold } \\
\text { Enrichment }\end{array}$ & FDR \\
\hline Deoxyribonucleotide Metabolism & 4 & 23.8 & $3.1 \times 10^{-2}$ \\
Innate Immunity & 25 & 5.4 & $2.3 \times 10^{-7}$ \\
\hline
\end{tabular}

b

\begin{tabular}{lrrr}
\hline Gene Ontology of $\mathbf{3 8 7}$ Targets Indirectly Downregulated by HIF-1 & \\
\hline Process & $\begin{array}{r}\text { Number } \\
\text { of Genes }\end{array}$ & $\begin{array}{r}\text { Fold } \\
\text { Enrichment }\end{array}$ & FDR \\
\hline Protein Dephosphorylation & 12 & 3.6 & $2.8 \times 10^{-2}$ \\
Multicellular Development & 10 & 27.9 & $2.2 \times 10^{-2}$ \\
Transcriptional Regulation & 4 & 18.1 & $2.2 \times 10^{-2}$ \\
Organelle Organization \& Biogenesis & 7 & 33.4 & $4.4 \times 10^{-5}$ \\
Signal Transduction & 10 & 28.8 & $1.8 \times 10^{-2}$ \\
Nucleic Acid Metabolism & 6 & 21.2 & $2.5 \times 10^{-2}$ \\
\hline
\end{tabular}




\section{Extended Data Figure 6. Vora et al.}

a

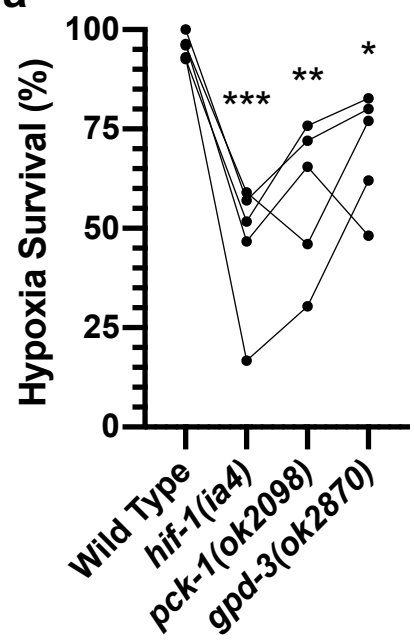

b

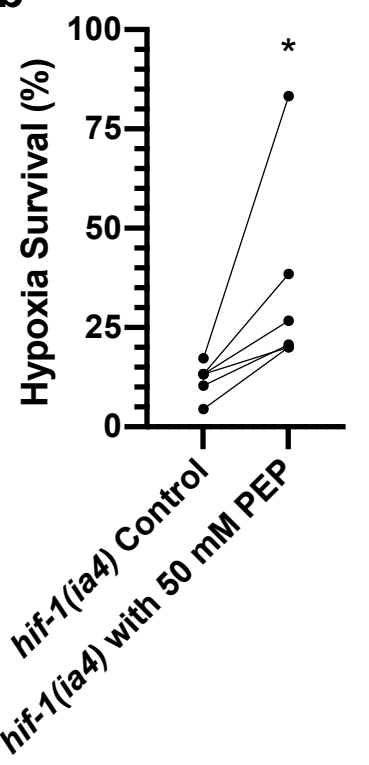

C

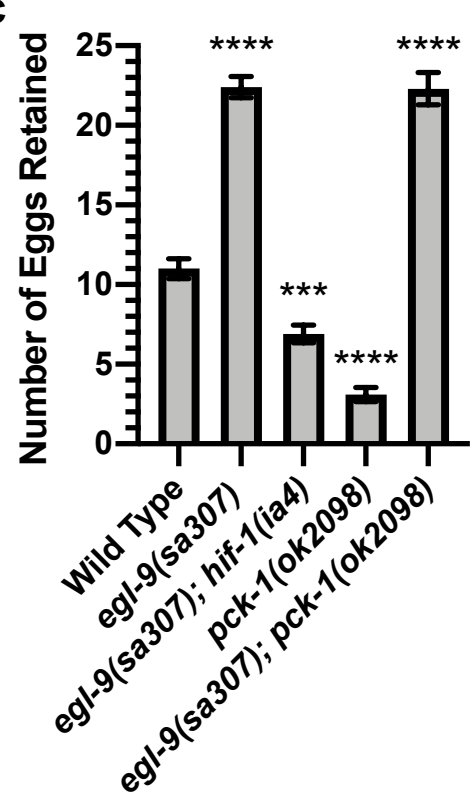

d

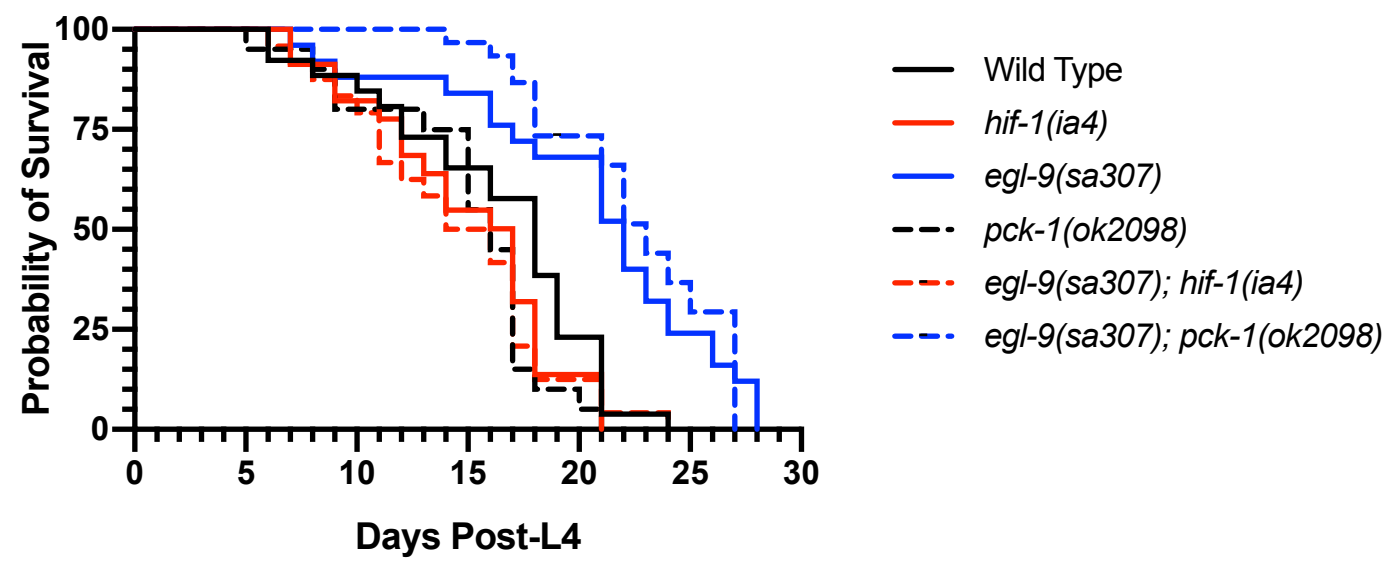

$\begin{array}{llll}\text { condition } & \text { chi } & \text { pvalue } & \text { FDR } \\ \text { Wild Type v.s. } \text { hif-1(ia4) } & 2.04 & 0.1535 & 0.7674 \\ \text { Wild Type v.s. egl-9(sa307) } & 12.65 & 0.0004 & 0.0019 \\ \text { Wild Type v.s. egl-9(sa307); hif-1(ia4) } & 2.04 & 0.1531 & 0.7655 \\ \text { Wild Type v.s. egl-9(sa307); pck-1(ok2098) } & 21.51 & 0.0000035 & 0.000018 \\ \text { Wild Type v.s. pck-1(ok2098) } & 1.09 & 0.2966 & 1 \\ \text { egl-9(sa307) v.s. egl-9(sa307); pck-1(ok2098) } & 0.27 & 0.605 & 1\end{array}$

\begin{tabular}{llll}
\hline condition & Censored & Deaths & Median Survival (Days) \\
\hline Wild Type & 4 & 26 & 18 \\
\hline hif-1(ia4) & 8 & 22 & 17 \\
\hline egl-9(sa307) & 5 & 25 & 22 \\
\hline pck-1(ok2098) & 0 & 20 & 16 \\
egl-9(sa307); hif-1(ia4) & 6 & 24 & 15 \\
egl-9(sa307); pck-1(ok2098) & 12 & 18 & 23
\end{tabular}

